\title{
Convection-pulsation coupling
}

\section{Excitation and stabilization mechanisms in $\delta$ Sct and $\gamma$ Dor stars}

\author{
M.-A. Dupret ${ }^{1}$, A. Grigahcène ${ }^{1}$, R. Garrido ${ }^{1}$, M. Gabriel ${ }^{2}$, and R. Scuflaire ${ }^{2}$ \\ 1 Instituto de Astrofísica de Andalucía-CSIC, Apartado 3004, 18080 Granada, Spain \\ e-mail: ahmed@iaa.es \\ 2 Institut d'Astrophysique et de Géophysique de l'Université de Liège, Belgium
}

Received 9 August 2004 / Accepted 23 January 2005

\begin{abstract}
We apply here the Time Dependent Convection (TDC) treatment presented in our earlier paper in this series to the study of $\delta$ Sct and $\gamma$ Dor pulsating stars. Stabilization of the $\delta$ Sct p-modes at the red edge of the Instability Strip (IS) and the driving of the $\gamma$ Dor g-modes are explained by our models. Theoretical IS obtained with different values of the Mixing Length (ML) parameter $\alpha$ are compared to observations and a good agreement is obtained for $\alpha$ between 1.8 and 2. The influence of each term of our TDC treatment (perturbation of convective flux, turbulent pressure, and turbulent kinetic energy dissipation) on the eigenfrequencies and on the driving and damping mechanisms is investigated. Finally, we show that our TDC models predict the likely existence of hybrid stars with both $\delta$ Sct p-modes and $\gamma$ Dor g-modes oscillations.
\end{abstract}

Key words. stars: oscillations - convection - stars: interiors - stars: variables: $\delta$ Sct - stars: variables: general

\section{Introduction}

$\delta$ Sct stars are variable A-F types stars located at the intersection of the Cepheid IS with the main sequence. Their pulsation periods go from 0.5 to $6 \mathrm{~h}$ and correspond to low order $\mathrm{p}$ - and g-modes. It is well known that $\kappa-\gamma$ mechanism in the HeII partial ionization zone is the main driving mechanism in this type of stars.

The theoretical blue edge of the $\delta$ Sct IS has been determined by many authors for radial, as well as for non-radial modes (e.g. Pamyatnykh 2000). But determining the theoretical red edge is a more difficult matter, because it requires a non-adiabatic treatment of the interaction between convection and pulsation (Baker \& Kippenhahn 1965). Houdek (2000) studied the convective effects on radial p-mode stability in $\delta$ Sct stars, using the time-dependent convection treatment of Gough (1977), together with the non-local treatment of Balmforth (1992). Xiong et al. (2001) and Xu et al. (2002) succeeded obtaining a theoretical red edge for radial modes, using the non-local time-dependent convection theory of Xiong et al. (1997). In this paper, we present theoretical blue and red edges of the $\delta$ Sct IS obtained for radial and non-radial modes as well, using the time-dependent convection (TDC) treatment presented in Grigahcène et al. (2004b, hereafter Paper I).

$\gamma$ Dor stars are a recently discovered class of variable stars with a range in spectral types of A7-F5 and of luminosity class IV-V, or V (Kaye et al. 1999). Their long periods (between 0.4 and 3 days) correspond to pulsations in non-radial high order gravity modes, but the driving mechanism of these g-modes is not yet fully understood. Using frozen convection (FC) models, Guzik et al. (2000) showed that the driving of the $\gamma$ Dor g-modes can be explained by a convective flux blocking mechanism at the base of their Convective Envelope (CE). Following this approach, a first theoretical IS has been obtained by Warner et al. (2003); however, in a significant part of the $\mathrm{CE}$, the FC approximation is not valid, because the lifetime of the convective elements becomes shorter than the pulsation period. Therefore, TDC computations are required to test the validity of the flux blocking mechanism. We show that the driving of the $\gamma$ Dor g-modes by this mechanism is successfully confirmed by our TDC models and present theoretical IS which are in good agreement with observations.

Preliminary determinations of theoretical IS for $\delta$ Sct and $\gamma$ Dor based on the TDC theory presented in Paper I are given in Dupret et al. (2004a,b) and Grigahcène et al. (2004a). In Sect. 2, we recall the role played by the different terms of this TDC treatment on the mode excitation and stabilization. Detailed applications to $\delta$ Sct and $\gamma$ Dor stars are presented in Sects. 3, 4, and 5. Finally, we show in Sect. 6 that our theoretical models predict the possible occurrence of simultaneous $\delta$ Sct p-modes and $\gamma$ Dor g-modes oscillations for the same stellar model.

\section{Time-Dependent Convection (TDC) treatment}

Details of the TDC treatment adopted in our study are given in Paper I. We recall that our TDC treatment, derived in the 
framework of the Mixing-Length Theory (MLT), takes the following into account: perturbations of the convective flux $\left(\delta F_{\mathrm{c}}\right)$, turbulent pressure $\left(\delta p_{\mathrm{t}}\right)$, the dissipation rate of turbulent kinetic energy into heat $\left(\delta \overline{\epsilon_{2}}\right)$, and the the power produced by the buoyancy force $\left(-\overline{\boldsymbol{V} \cdot \boldsymbol{\nabla} p_{\mathrm{th}}}\right) \cdot \delta F_{\mathrm{c}}$ appears in the equation of total energy conservation; $\delta p_{\mathrm{t}}$ appears in the equation of momentum conservation; and $\delta\left(\overline{\epsilon_{2}+\boldsymbol{V} \cdot \boldsymbol{\nabla} p_{\text {th }}}\right)$ appears both in the equation of total energy conservation and in the equation of turbulent kinetic energy conservation but with opposite sign. Each of these terms plays a specific role in the excitation and damping of the modes. More precisely, we showed in Paper I that the total work integral is $W=W_{f_{\mathrm{R}}}+W_{f_{\mathrm{c}}}+W_{p_{\mathrm{t}}}+W_{\epsilon_{2}}$ (we do not consider here the effect of nuclear reactions on the mode excitation), where for a radial mode and isotropic turbulence,

$W_{f_{\mathrm{R}}}=-\int_{0}^{m}\left(\Gamma_{3}-1\right) \mathfrak{R}\left\{\frac{1}{\sigma} \frac{\delta \rho^{*}}{\rho} \frac{\mathrm{d} \delta L_{\mathrm{R}}}{\mathrm{d} m}\right\} \mathrm{d} m$

is the work due to radiative luminosity perturbation;

$W_{f_{\mathrm{c}}}=-\int_{0}^{m}\left(\Gamma_{3}-1\right) \mathfrak{R}\left\{\frac{1}{\sigma} \frac{\delta \rho^{*}}{\rho} \frac{\mathrm{d} \delta L_{\mathrm{c}}}{\mathrm{d} m}\right\} \mathrm{d} m$

is the work due to convective luminosity perturbation;

$W_{p_{\mathrm{t}}}=-\int_{0}^{m} \mathfrak{J}\left\{\frac{\delta \rho^{*}}{\rho} \frac{\delta p_{\mathrm{t}}}{\rho}\right\} \mathrm{d} m$

is the work due to the perturbation of the turbulent pressure; and

$$
\begin{aligned}
W_{\epsilon_{2}} & \left.=\int_{0}^{m}\left(\Gamma_{3}-1\right) \mathfrak{R}\left\{\frac{1}{\sigma} \frac{\delta \rho^{*}}{\rho} \delta \overline{\epsilon_{2}+\boldsymbol{V} \cdot \frac{\nabla p_{\mathrm{th}}}{\rho}}\right)\right\} \mathrm{d} m \\
& =\int_{0}^{m} \frac{3}{2}\left(\Gamma_{3}-1\right) \mathfrak{J}\left\{\frac{\delta \rho^{*}}{\rho} \frac{\delta p_{\mathrm{t}}}{\rho}\right\} \mathrm{d} m
\end{aligned}
$$

is the work due to turbulent kinetic energy dissipation variations.

For non-radial modes, the transversal components of the flux perturbation also play a role in the work integral. Using the notation

$\delta \boldsymbol{F}_{i}=\delta F_{i, r}(r) Y_{\ell}^{m}(\theta, \phi) \boldsymbol{e}_{r}+\delta F_{i, h}(r)\left(r \boldsymbol{\nabla}_{\boldsymbol{h}} Y_{\ell}^{m}(\theta, \phi)\right)$

where $i=\mathrm{c}$ for convection and $i=\mathrm{R}$ for radiation, the contributions of the radiative and convective flux transversal components to the work integral are:

$W_{\mathrm{f}_{i, h}}=\ell(\ell+1) \int_{0}^{m}\left(\Gamma_{3}-1\right) \mathfrak{R}\left\{\frac{\delta \rho^{*} F_{i}}{r \rho^{2} \sigma}\left[\frac{\delta F_{i, h}}{F_{i}}-\frac{\xi_{h}}{r}\right]\right\} \mathrm{d} m$.

The equilibrium stellar models were computed with the evolutionary code CLÉS (Code Liégeois d'Évolution Stellaire) that uses: standard MLT for convection calculations; the OPAL opacities (Iglesias \& Rogers 1996) completed at low temperatures with the opacities of Alexander \& Ferguson (1994); the CEFF equation of state (Christensen-Dalsgaard \& Däppen 1992); and the atmosphere models of Kurucz (1998) as boundary conditions. All the models of this study have a chemical composition given by: $(X=0.7, Z=0.02)$ and a convective core overshooting parameter $\alpha_{\mathrm{ov}}=0.2$. In the present study, we used more than 10000 models, and in Table 1, we give the global parameters of the structure models we used to determine the eigenfunctions presented in this paper.
Table 1. Global parameters of the models.

\begin{tabular}{cccccc}
\hline \hline & $M / M_{\odot}$ & $\log \left(L / L_{\odot}\right)$ & $T_{\text {eff }}$ & $\log (g)$ & $\alpha$ \\
\hline Model 1 & 1.8 & 1.1988 & 6681 & 3.7467 & 1.8 \\
Model 2 & 1.6 & 0.9731 & 6665 & 3.9172 & 1.8 \\
Model 3 & 1.7 & 1.0615 & 7267 & 4.0055 & 1 \\
Model 4 & 1.8 & 1.1421 & 7824 & 4.0779 & 1 \\
Model 5 & 1.6 & 0.9564 & 6935 & 4.0028 & 2 \\
Model 6 & 1.6 & 0.9829 & 6443 & 3.8485 & 2 \\
Model 7 & 1.6 & 0.9399 & 7086.5 & 4.0569 & 1.8 \\
Model 8 & 1.6 & 0.9552 & 6931 & 4.0031 & 1.8 \\
\hline
\end{tabular}

\section{3. $\delta$ Sct stars}

We present in the following subsections application of our TDC treatment to the study of the excitation and damping mechanisms of $\delta$ Sct stars. In Sects. 3.1, 3.2, 3.3 and, when not otherwise specified, the prescriptions of our TDC treatment are the following: (i) only the perturbation of the convective flux is taken into account; (ii) Eq. (27) of Paper I is used for perturbation of the mixing-length; (iii) parameter $\beta$ introduced in Sect. 3.2 of Paper I is equal to zero. We begin by showing in Sect. 3.1 that the stabilization of the modes in cold models is obtained with our TDC treatment but not with FC. Nonadiabatic computations for a series of models with different masses have allowed us to find the theoretical IS of the $\delta$ Sct stars for radial and non-radial modes. The results for models with $\alpha=1.8$ are presented in Sect. 3.2. Our results depend on parameters of both the equilibrium and perturbed model. First, in Sect. 3.3 we discuss the sensitivity of the theoretical IS to the value of ML parameter $\alpha$. Secondly, in Sect. 3.4 we compare the results obtained with different expressions for the perturbation of mixing length $l$. In Sect. 3.5, the physical interpretation of our results is considered by more closely analysing the role played by each term of our TDC treatment on the excitation and damping mechanisms occurring at the blue and red sides of the IS. And finally in Sect. 3.6, we discuss the occurrence of short wavelength oscillations in the eigenfunctions for models close to the IS red edge and we show the results obtained with a new local TDC treatment which allows them to be avoided.

\subsection{Mode stabilization}

In this section, we show that stabilization of radial and nonradial modes at the red edge of the IS is obtained with our TDC treatment. The comparison to observations and the analysis of the main sources of damping and driving will be done in the next sections. In Fig. 1, we give the evolution from ZAMS to TAMS of the dimensionless frequencies $\omega$ of $\ell=0$ and $\ell=2$ modes as a function of the logarithm of the effective temperature for $1.8 M_{\odot}$ models with a ML parameter $\alpha=1.8$ (solar calibrated value for our evolutionary code). $\omega=\sigma t_{\mathrm{dyn}}$, where $\sigma$ is the angular frequency and $t_{\mathrm{dyn}}=\sqrt{R^{3} / G M}$ is the dynamical time of the star. Top and middle left panels of this figure show the evolution of dimensionless frequencies in the FC approximation. It is seen that FC models do not lead to 
$\mathrm{l}=0 \mathrm{FC}$

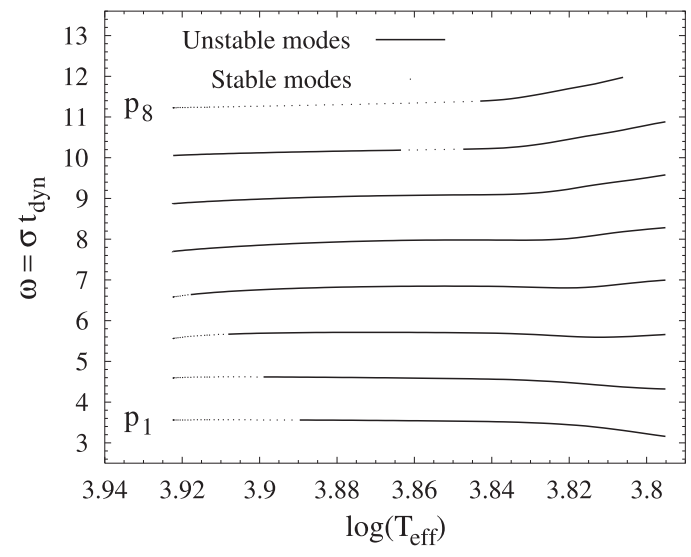

$1=2 \mathrm{FC}$

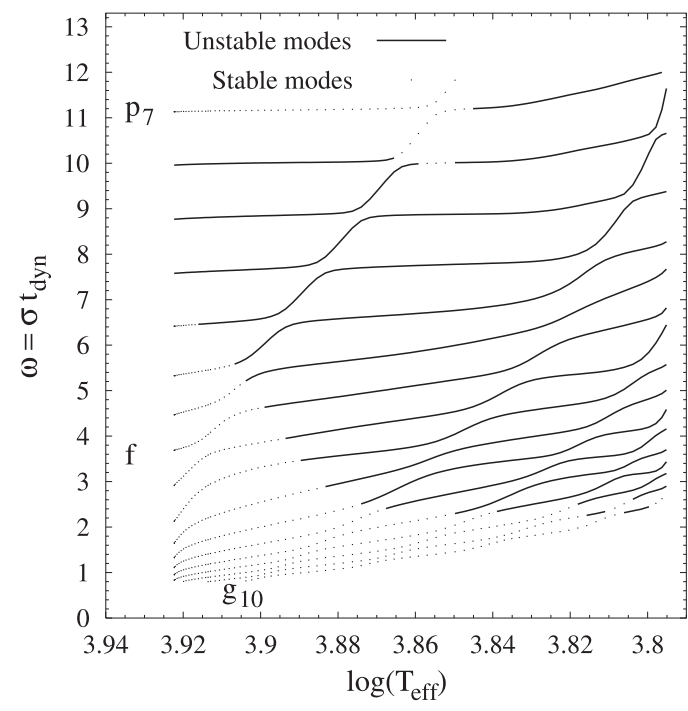

$\mathrm{l}=0 \mathrm{TDC} \delta \mathrm{l} / \mathrm{l}=\delta \mathrm{H}_{\mathrm{p}} / \mathrm{H}_{\mathrm{p}}$

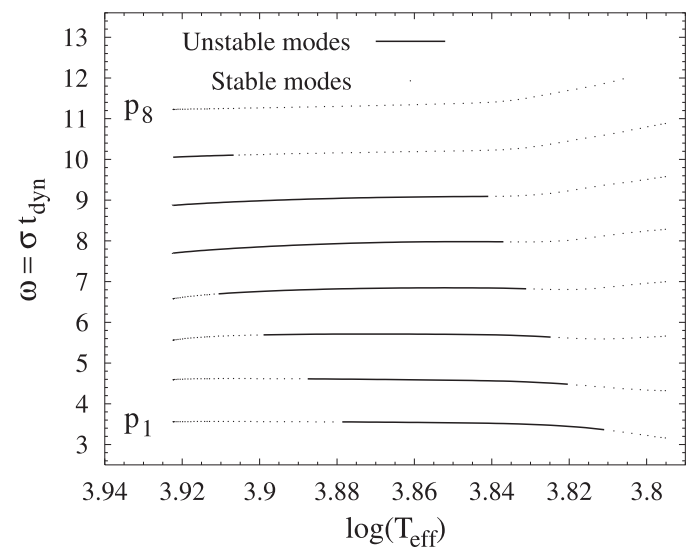

$\mathrm{l}=0 \mathrm{TDC}$

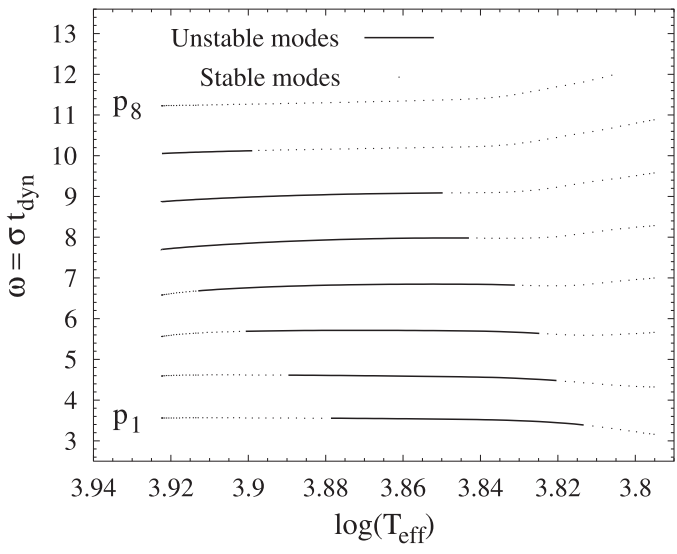

$1=2$ TDC

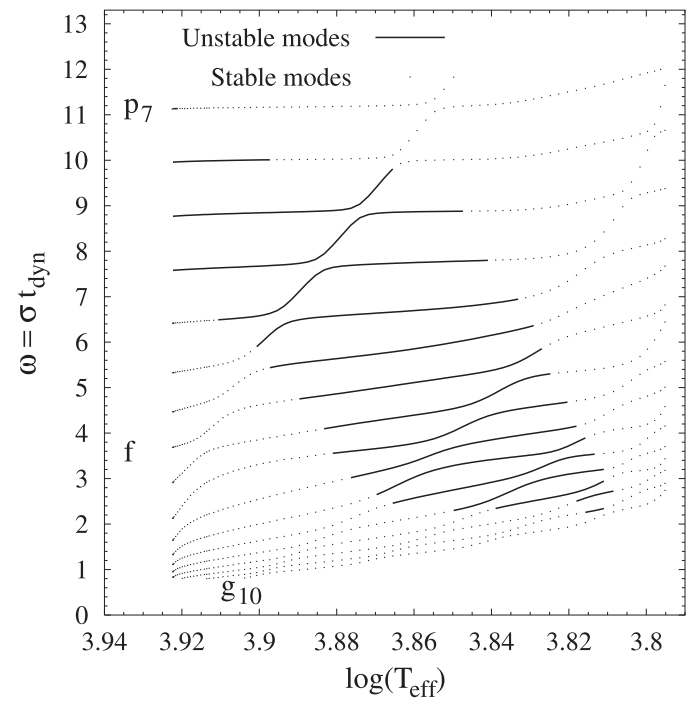

$1=0$ TDC $\beta=1$

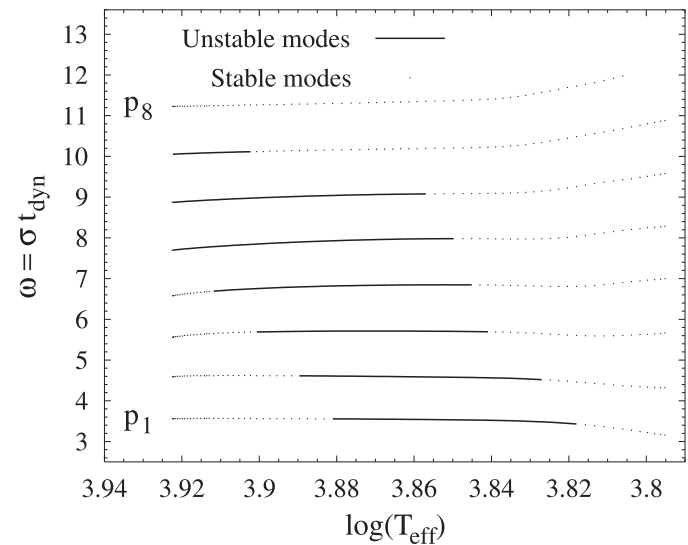

Fig. 1. Evolution of the dimensionless frequencies of the stable and unstable modes for stellar models of $1.8 M_{\odot}$ with $\alpha=1.8$. Top and middle left panels correspond to FC results and other panels correspond to TDC results taking only the perturbation of convective flux into account. Top panels and bottom panels correspond to $\ell=0$ modes and middle panels to $\ell=2$ modes. In the three right panels, we assume $\delta l / l=\left(\delta H_{\mathrm{p}} / H_{\mathrm{p}}\right) /\left(1+\left(\sigma \tau_{\mathrm{c}}\right)^{2}\right)$ and in bottom left panel, we assume $\delta l / l=\delta H_{\mathrm{p}} / H_{\mathrm{p}}$. In the bottom right panel we assume $\beta=1$ and in the other panels $\beta=0$. Stabilization at the $\delta$ Sct red edge is obtained with our TDC models but not with FC models.

stabilizing the modes at low temperatures. All the other panels of this figure show that, on the contrary, our TDC models succeed in stabilizing the radial and non-radial modes at low temperatures, leading therefore to an IS red edge. The typical range of unstable modes for $\ell=0,1,2$, and 3 modes is given in Table 2. The frequency range of the unstable modes predicted 
Table 2. Range of unstable modes predicted by our $1.8 M_{\odot}$ models, for modes of degree $\ell=0,1,2$, and 3. $n_{\max }$ (resp. $n_{\min }$ ) gives the radial order of the unstable mode of highest (resp. lowest) frequency at the blue side (resp. red side) of the IS.

\begin{tabular}{lcccc}
\hline \hline & $\ell=0$ & $\ell=1$ & $\ell=2$ & $\ell=3$ \\
\hline$n_{\max }$ (blue side) & $\mathrm{p}_{7}$ & $\mathrm{p}_{7}$ & $\mathrm{p}_{7}$ & $\mathrm{p}_{6}$ \\
$n_{\min }$ (red side) & $\mathrm{p}_{1}$ & $\mathrm{~g}_{6}$ & $\mathrm{~g}_{9}$ & $\mathrm{~g}_{11}$ \\
\hline
\end{tabular}

by our models is in agreement with the frequencies typically observed for $\delta$ Sct stars.

\subsection{Theoretical instability strips}

In order to determine theoretical IS, a large number of evolutionary tracks were computed with masses ranging from 1.4 to $2.2 M_{\odot}$, and with a step of $0.05 M_{\odot}$. Performing non-adiabatic pulsation computations for all these stellar models, we determined the theoretical IS for $\delta$ Sct stars obtained with our TDC treatment. In Fig. 2, we present the theoretical IS obtained for radial modes for models with $\alpha=1.8$. Each curve corresponds to the blue or red edge of a mode of given radial order $n$. Labels enable us to identify the modes (e.g. $\mathrm{p}_{6 \mathrm{R}}$ for the red edge of the $\mathrm{p}_{6}$ mode). As the radial order of the modes increases, the blue and red edges are shifted towards higher $T_{\text {eff }}$. The small points correspond to the position of the 636 observed $\delta$ Sct stars of the Rodriguez et al. (2000) catalogue, for which effective temperatures were obtained using the calibrations of Moon \& Dworetsky (1985). For comparison, we also give in Fig. 2 the position of the theoretical red edge for the fundamental radial mode obtained by Xiong et al. (2001) (“`”) and by Houdek (2000) (“•”). Xiong's red edge is colder than our red edge, while Houdek's red edge is hotter than our red edge, which could come from the higher $\alpha=2$ value adopted in Houdek's models. From a theoretical point of view, Xiong's theory is more elaborate than those based on MLT. However, his complex theory also requires static models to be computed with this more elaborate theory than the usual one in evolution codes, MLT, which we must use for sake of consistency. Houdek uses a different MLT than ours and also neglects the term in $W_{\epsilon_{2}}$, which as we will see is, at least for the stars considered in this paper, of the same order as $W_{p_{\mathrm{t}}}$. We also note that he chooses large values for parameters " $a$ " and " $b$ " $\left(a^{2}=b^{2}=600\right)$ in his non-local treatment. The 3 theories are very different from each other and can be compared through their numerical result as is done in Fig. 2, while our TDC theory is the only one which applies to non-radial oscillations.

We also obtained theoretical IS for the $\delta$ Sct non-radial modes. In Fig. 3, we present the results obtained for $\ell=2$ modes for models with $\alpha=1.8$. Again we see that, as the radial order of the modes increases, the blue and red edges are displaced towards higher $T_{\text {eff }}$. For the sake of clarity in the figure, we only give the results for $\mathrm{g}_{3}$ to $\mathrm{p}_{4}$ modes. For non-radial modes, the shapes of the blue and red edges are not as straight as for radial modes, due to the avoided crossings that can be seen in the middle panels of Fig. 1.

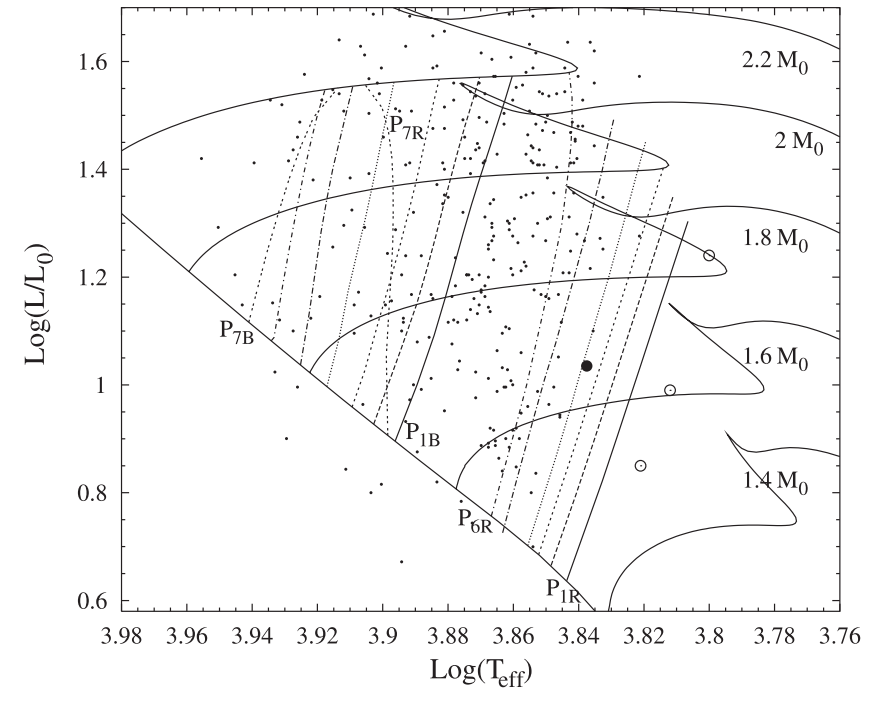

Fig. 2. Blue and red edges of the $\delta$ Sct theoretical IS. Lines are our TDC results for radial modes from $\mathrm{p}_{1}$ to $\mathrm{p}_{7}$ for models with $\alpha=1.8$. The small points correspond to observations. For comparison, we also give the red edges for the fundamental radial mode obtained by Xiong et al. (2001) (“॰”) and by Houdek (2000) (“•”).

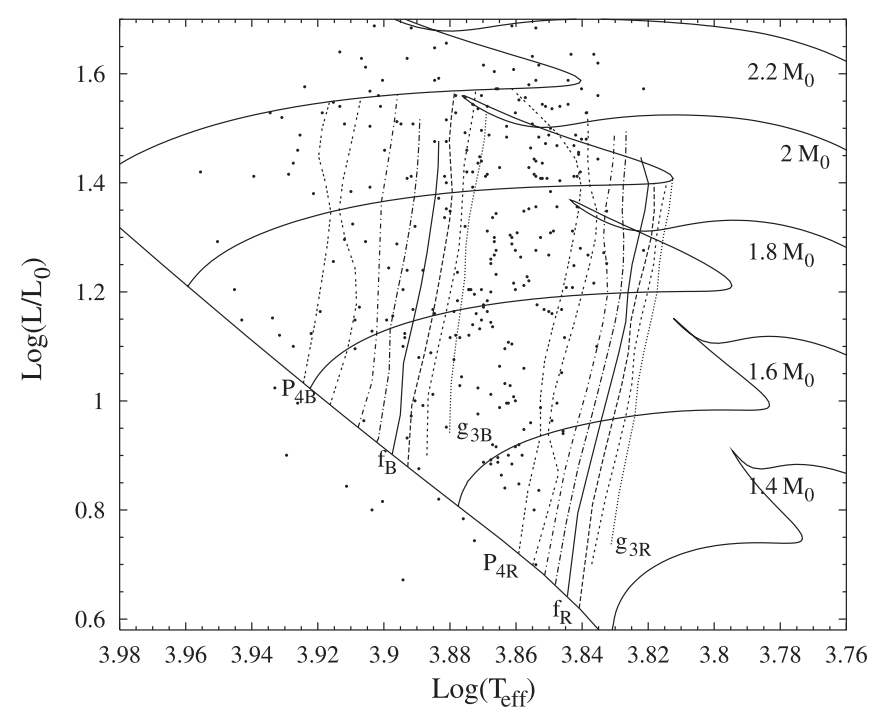

Fig. 3. Blue and red edges of the $\delta$ Sct theoretical IS for models with $\alpha=1.8$ and $\ell=2$ modes from $\mathrm{g}_{3}$ to $\mathrm{p}_{4}$, as obtained with our TDC treatment.

\subsection{Influence of the $M L$ parameter $\alpha$}

To study the sensitivity of the theoretical IS to the value of the ML parameter $\alpha$, we give the theoretical IS for radial modes and for models with $\alpha=1$ in Fig. 4. For the sake of clarity, we only give results for modes from $\mathrm{p}_{1}$ to $\mathrm{p}_{4}$, where we notice that the theoretical red edges are displaced towards lower effective temperatures when $\alpha$ decreases. The physical reason is that the red edge corresponds to models with a given size of the CE. With lower $\alpha$, the CE is thinner for a given $T_{\text {eff }}$. Therefore, we must go to smaller $T_{\text {eff }}$ to have a CE sufficiently deep to stabilize the modes. Comparing Figs. 2 and 4 shows that we get better agreement with observations for the solar calibrated value $\alpha=1.8$. A preliminary study of influence of the metallicity 


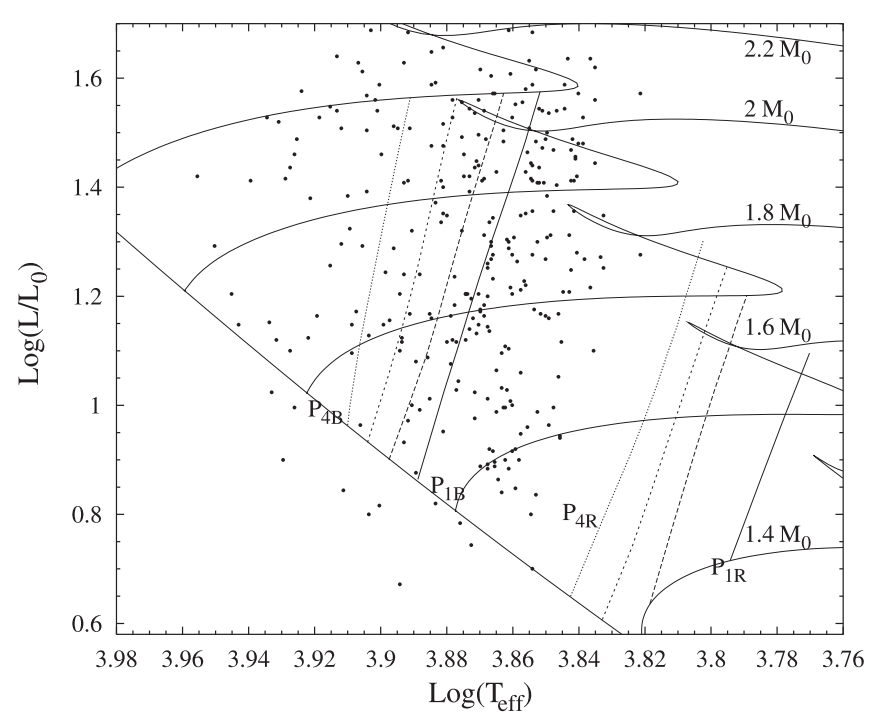

Fig. 4. Blue and red edges of the $\delta$ Sct theoretical IS for models with $\alpha=1$ and $\ell=0$ modes from $\mathrm{p}_{1}$ to $\mathrm{p}_{4}$, as obtained with our TDC treatment.

and overshooting shows that they affect the IS red edge location much less than $\alpha$, but this will be analyzed more closely in future work.

\subsection{Influence of the mixing-length perturbation}

A source of uncertainty in any perturbation of MLT is in the way to perturb the mixing length $l$. In Paper I (Sect. 2.4), we mentioned different ways to perturb this quantity. In the top and middle right panels of Fig. 1, we show the stable and unstable radial and the $\ell=2$ modes obtained adopting $\delta l / l=\left(\delta H_{\mathrm{p}} / H_{\mathrm{p}}\right) /\left(1+\left(\sigma \tau_{\mathrm{c}}\right)^{2}\right)$. In the bottom left panel of Fig. 1, we give results obtained for radial modes, now adopting $\delta l / l=\delta H_{\mathrm{p}} / H_{\mathrm{p}}$. We see that these two ways to perturb $l$ give nearly the same results for $\delta$ Sct stars. More precisely, the differences between the two red edges are negligible for the modes $\mathrm{p}_{1}$ to $\mathrm{p}_{4}$, while the effective temperature shift between the two red edges is about $100-150 \mathrm{~K}$ for the modes $\mathrm{p}_{5}$ to $\mathrm{p}_{7}$. The results are much more sensitive to the value adopted for the ML parameter $\alpha$, as shown in the previous section.

\subsection{Excitation and damping mechanisms}

In this section, we analyse the excitation and damping mechanisms in $\delta$ Sct stars in more details. First, at the blue side of the IS, the $\kappa$-mechanism in the HeII partial ionization zone plays a major role, and the CE is very thin. For hot models, the difference between FC and TDC works is thus negligible. But for colder models, where the CE extends deeper, TDC plays a significant role in the excitation and damping of the modes. In Fig. 5, we give the work integrals for the radial mode $\mathrm{p}_{3}$ and a model at the IS red edge and give the results obtained including $\delta \boldsymbol{F}_{\mathbf{c}}, \delta p_{\mathrm{t}}$ and $\delta \epsilon_{2}(6)$. We also give the contribution of each of these 3 terms in the total work and the result obtained with FC treatment. The work integrals are normalized so that their surface value is the dimensionless growth rate $(-\mathfrak{J}(\omega))$ that

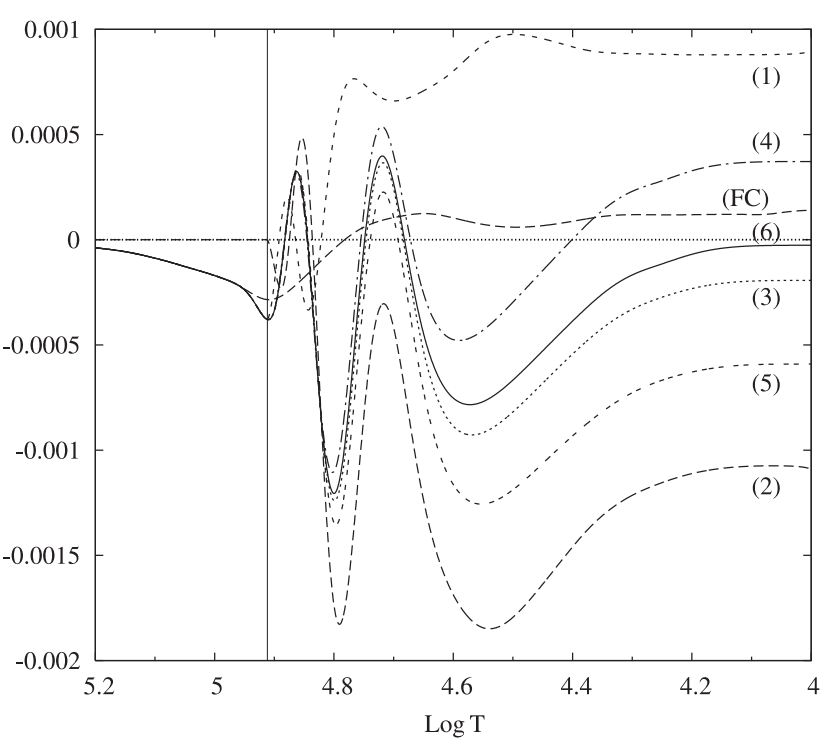

Fig. 5. Different physical components of the work integral obtained with FC and TDC treatment for the radial $\mathrm{p}_{3}$ mode of Model 1 of Table 1. (1) is $W_{f_{\mathrm{R}}} ;(2)$ is $W_{f_{\mathrm{c}}} ;(3)$ is $W_{f_{\mathrm{R}}}+W_{f_{\mathrm{c}}}$; (4) is $W_{f_{\mathrm{R}}}+W_{f_{\mathrm{c}}}+W_{p_{\mathrm{t}}}$; (5) is $W_{f_{\mathrm{R}}}+W_{f_{\mathrm{c}}}+W_{\epsilon_{2}} ;(6)$ is $W=W_{f_{\mathrm{R}}}+W_{f_{\mathrm{c}}}+W_{p_{\mathrm{t}}}+W_{\epsilon_{2}}$, and (FC) is the work integral obtained with FC treatment. On the IS red side, the perturbed convection terms play a significant role in all the CE. $W_{p_{\mathrm{t}}}$ and $W_{\epsilon_{2}}$ have the opposite effect on the mode excitation.

is positive for unstable modes and negative for stable modes. Regions where the work integral increases (or decreases) are driving (or damping) the oscillations.

We see in Fig. 5 that $W_{f_{\mathrm{R}}}$ (Curve 1) has a driving effect at the $\mathrm{CE}$ base due to a flux blocking mechanism that will be explained in detail in Sect. 4.3 for $\gamma$ Dor stars. The same flux blocking can be seen in the FC result. We also notice small variations of $W_{f_{\mathrm{R}}}$ inside the CE below $\log T=4.8$, which is due to $\kappa$-mechanism in the $\mathrm{He}$ and $\mathrm{H}$ partial ionization zones. However, the radiative luminosity $L_{\mathrm{R}}$ is already strongly reduced in these regions so that the $\kappa$-mechanism that acts through $L_{\mathrm{R}}$ can no longer be efficient in driving the pulsation in the coldest $\delta$ Scuti stars. In these stars the main driving comes from the flux blocking mechanism which, for these modes, is efficient enough to make all those stars with an adequately deep convection zone unstable in the FC approximation. This explains why no red edge of the IS is found in the FC case.

However, for $\delta$ Sct stars, $W_{f_{\mathrm{c}}}$ (Curve 2 of Fig. 5) is significant in all CE and compensates for the driving effect of $W_{f_{\mathrm{R}}}$ (as shown in Curve $3=W_{f_{\mathrm{R}}}+W_{f_{\mathrm{c}}}$ ), so that the stabilization of the modes at the red edge of the IS is obtained with TDC models. $W_{p_{\mathrm{t}}}$ (difference between Curve 4 and Curve 3 ) has a driving effect in this model. But it is nearly compensated for by $W_{\epsilon_{2}}$ (difference between Curve 5 and Curve 3 ) which has a damping effect on the oscillations. Therefore $W_{f_{\mathrm{R}}}+W_{f_{\mathrm{c}}}(3)$ and $W(6)$ are not very different. 


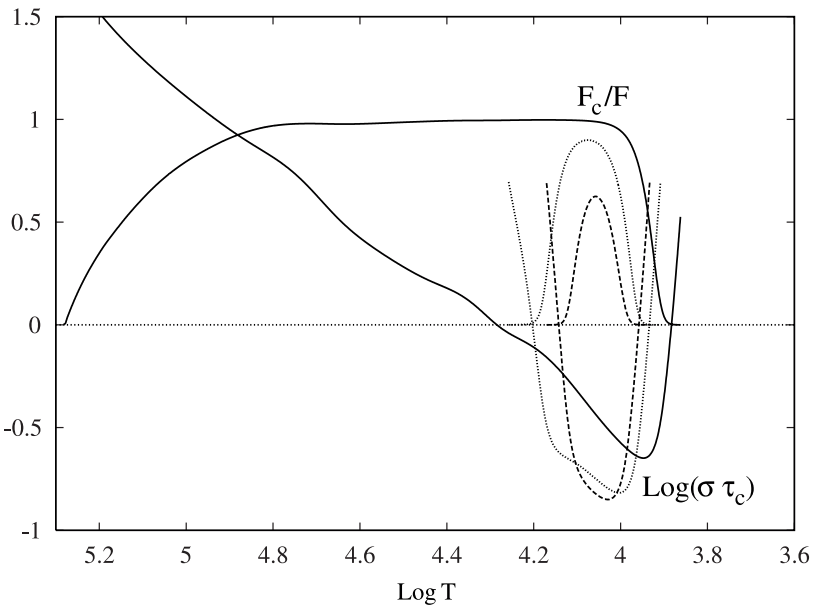

Fig. 6. $\log \left(\sigma \tau_{\mathrm{c}}\right)$ (fundamental radial mode) and $F_{\mathrm{c}} / F$ for Model 2 (solid line), Model 3 (dotted line) and Model 4 (dashed line) of Table $1 . \log \left(\sigma \tau_{\mathrm{c}}\right)$ is truncated near the convective zones boundaries. For the two hot models, $\sigma \tau_{\mathrm{c}}<1$ in the CE; for the cold model, $\sigma \tau_{\mathrm{c}}>1$ in a significant part of the $\mathrm{CE}$.

\subsection{Short wavelength oscillations of the eigenfunctions}

As discussed in Paper I Sect. 3.1, short wavelength spatial oscillations of the thermal eigenfunctions can arise with our TDC treatment in the regions where $\sigma \tau_{\mathrm{c}} \gg 1$. In Fig. 6, we give values of $\log \left(\sigma \tau_{\mathrm{c}}\right)$ in the $\mathrm{CE}$ for the fundamental radial mode of $3 \delta$ Sct models with different effective temperatures. In Models 3 and 4 of Table 1, convection is inefficient, $\sigma \tau_{\mathrm{c}}<1$, and spatial oscillations are not present in the non-adiabatic solutions. These models are representative of most $\delta$ Sct stars; however, for a model near the $\delta$ Sct red edge (Model 2 of Table 1), the CE size is larger and $\sigma \tau_{\mathrm{c}}>1$ in most of it. Therefore oscillations of the eigenfunctions occur in a large fraction of the $\mathrm{CE}$, which can be seen in the top panel of Fig. 7, where we give the work integral of the fundamental radial mode obtained with TDC for this last model. The vertical line indicates the base of CE. In Sect. 3.2 of Paper I, we proposed a new local treatment that avoids these oscillations. In this new treatment, a free parameter $\beta$ of the order of unity is introduced. In the bottom panel of Fig. 7, we present the work integral obtained adopting the value $\beta=1$. We see that the short wavelength oscillations of the eigenfunctions disappear. For this model, we have $\mathfrak{J}(\omega)=7.3 \mathrm{E}-7$ with $\beta=1$, while $\mathfrak{J}(\omega)=2 . \mathrm{E}-7$ with $\beta=0$. In the bottom right panel of Fig. 1, we show the radial modes stabilization obtained with $\beta=1$. Comparing it to the top right panel of Fig. 1 shows that the stabilization occurs at similar effective temperatures for $\beta=0$ and $\beta=1$ models. This parameter, therefore, does not significantly influence the stability of the $\delta$ Sct models, but only prevents from the short wavelength oscillations. We notice that non-local solutions to the problem arising from these oscillations have also been proposed (Gonczi 1986; Balmforth 1992; Xiong et al. 1997). A comparison between the results obtained with local and non-local TDC treatments will be done in a future paper.
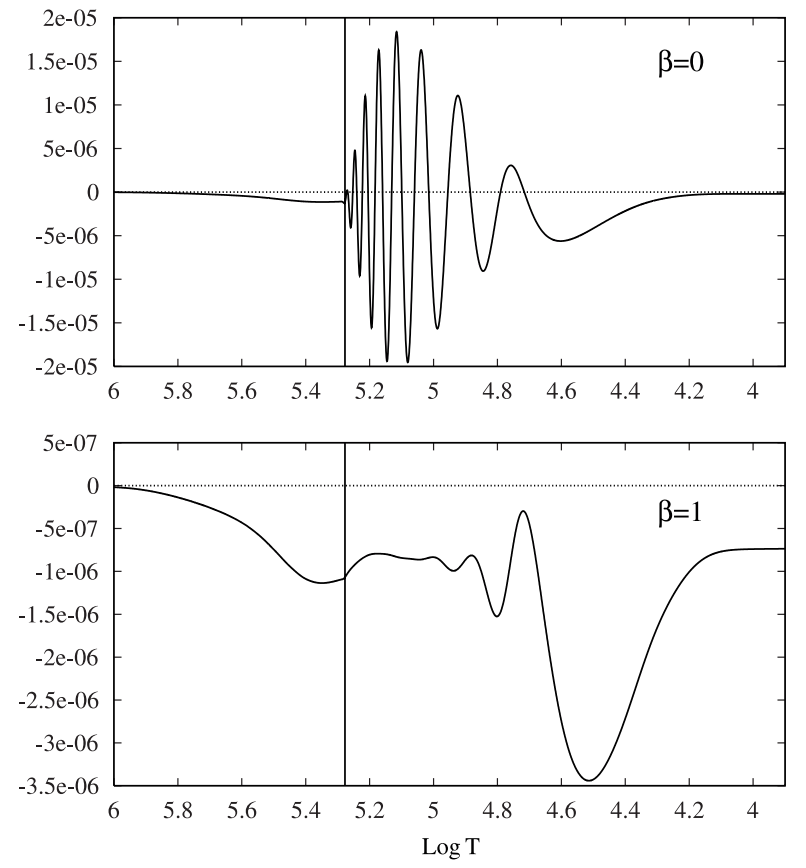

Fig. 7. Work integral obtained with our TDC treatment with $\beta=0$ (top panel) and with $\beta=1$ (bottom panel), for the fundamental radial mode. The vertical line indicates the base of CE. Model 2 of Table 1. The short wavelength spatial oscillations appearing with $\beta=0$ disappear with $\beta=1$.

\section{4. $\gamma$ Dor stars}

We now apply our TDC treatment to $\gamma$ Dor stars. As in the previous sections, when not specified, the prescriptions of our TDC treatment are the following: only the perturbation of the convective flux is taken into account; Eq. (27) of Paper I is used for the perturbation of the mixing-length; and the parameter $\beta$ introduced in Sect. 3.2 of Paper I is set to zero. In Sect. 4.1, we show the periods range of the unstable g-modes obtained with our TDC models. In Sect. 4.2, we present the theoretical IS obtained for different values of $\alpha$ and compare them to observations. And finally, in Sects. 4.3 and 4.4, we analyse the excitation and damping mechanisms of the $\gamma$ Dor g-modes in more detail.

\subsection{Unstable gravity modes}

Our TDC models are able to predict the driving of high order g-modes for typical models of $\gamma$ Dor stars. In Fig. 8, we show the periods range of the unstable modes predicted by our TDC models as a function of the effective temperatures for main sequence models of $1.6 M_{\odot}$. Each cross corresponds to an unstable mode. Results are given for all the modes with pulsation constant $Q=P t_{\mathrm{dyn}, \odot} / t_{\mathrm{dyn}} \geq 0.04$ days. $\delta$ Sct type unstable modes of smaller periods are also obtained but not given in this figure. We begin with the results for $\ell=1$ modes in the top panels, and compare the results obtained with $\alpha=2$ in the top left panel and with $\alpha=1.5$ in the top right panel. In both cases, the periods range of the unstable g-modes of our models is in agreement with the typical observed periods of $\gamma$ Dor stars. 

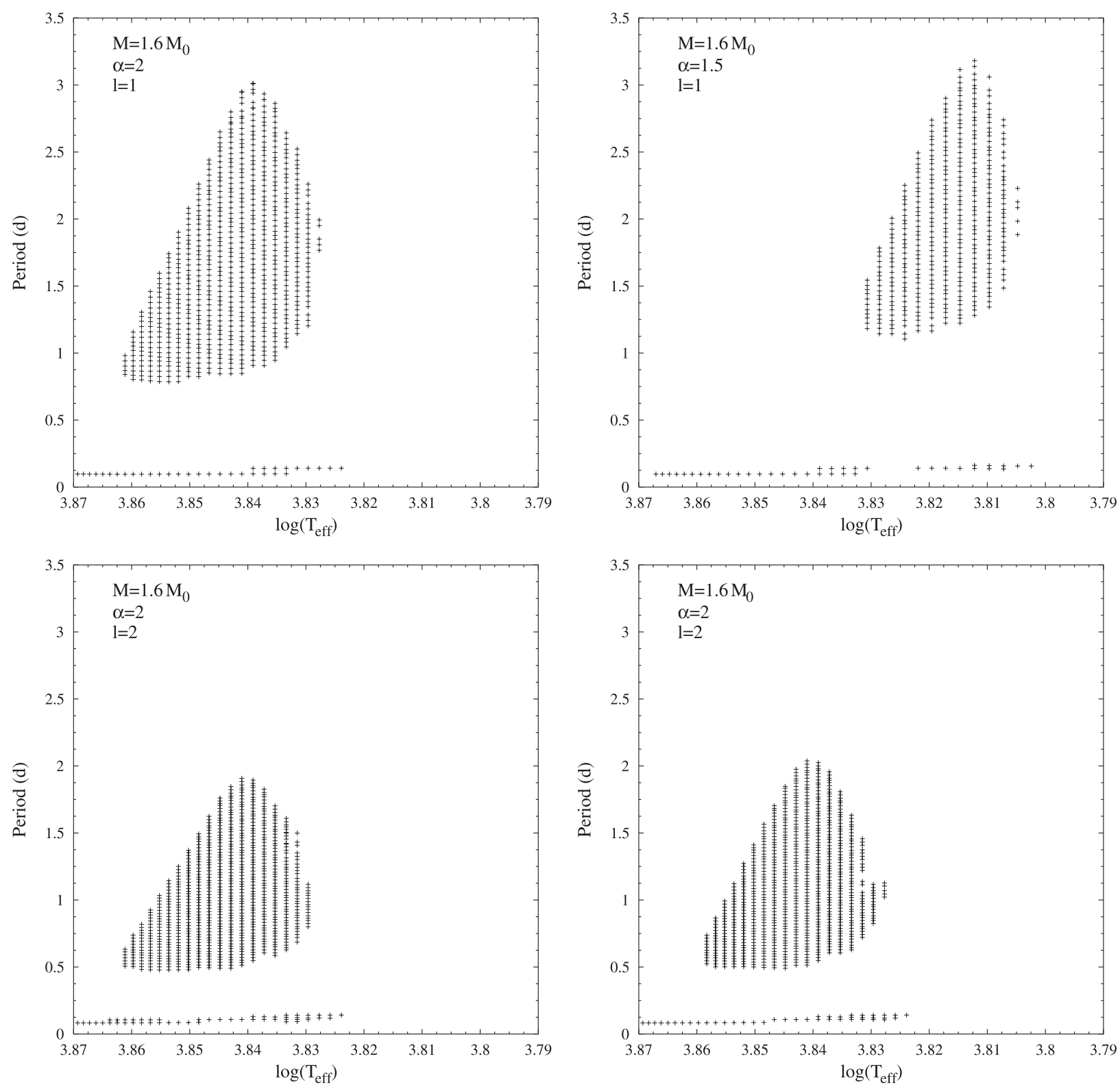

Fig. 8. Periods (in days) of the unstable gravity modes as a function of $\log \left(T_{\text {eff }}\right)$ obtained for TDC models of $1.6 M_{\odot}$. Each cross corresponds to a given mode. Top left panel is for $\ell=1$ modes and $\alpha=2$ models. The top right panel is for $\ell=1$ modes and $\alpha=1.5$ models. Bottom panels are for $\ell=2$ modes and $\alpha=2$ models. In bottom left panel only $\delta \boldsymbol{F}_{\mathrm{c}}$ is taken into account, while in bottom right panel, $\delta p_{t}$ and $\delta \epsilon_{2}$ are also taken into account. At the bottom of these panels, the unstable modes with $P \leq 0.2$ days correspond to $\delta$ Sct-type modes. The $T_{\text {eff }}$ and periods of unstable g-modes obtained with $\alpha=2$ models agree with typical observations of $\gamma$ Dor stars.

We see that the results are very sensitive to the adopted value of the ML parameter $\alpha$. With $\alpha=2$, the $T_{\text {eff }}$ range of unstable gmodes models is in better agreement with observations (Kaye et al. 1999) than for models with lower $\alpha$.

In the bottom panels, we give the results obtained for $\ell=2$ modes. We see that the periods of unstable $\ell=2$ modes are lower than those of $\ell=1$ modes. This could be expected since for g-modes the eigenvalue problem is essentially a function of the parameter $\ell(\ell+1) / \omega^{2}$. A similar dependence of the unstable g-modes periods on $\ell$ is also found in Slowly Pulsating B stars (Dupret 2002). Finally, we see that results that take only the perturbation of convective flux into account (bottom left panel of Fig. 8) are very similar to results where perturbation of turbulent pressure and turbulent kinetic energy dissipation are also taken into account (bottom right panel of Fig. 8).

\subsection{Theoretical instability strips}

In Fig. 9, we show the theoretical IS of $\gamma$ Dor $\ell=1$ modes obtained with our TDC treatment (thick lines) for three families of models with different values of the ML parameter $\alpha$ : $1,1.5$ and 2. For comparison, in the same figure we give the FC results of Warner et al. (2003) obtained with $\alpha=1.87$ (thin dashed lines). In this case, we give global IS and not 


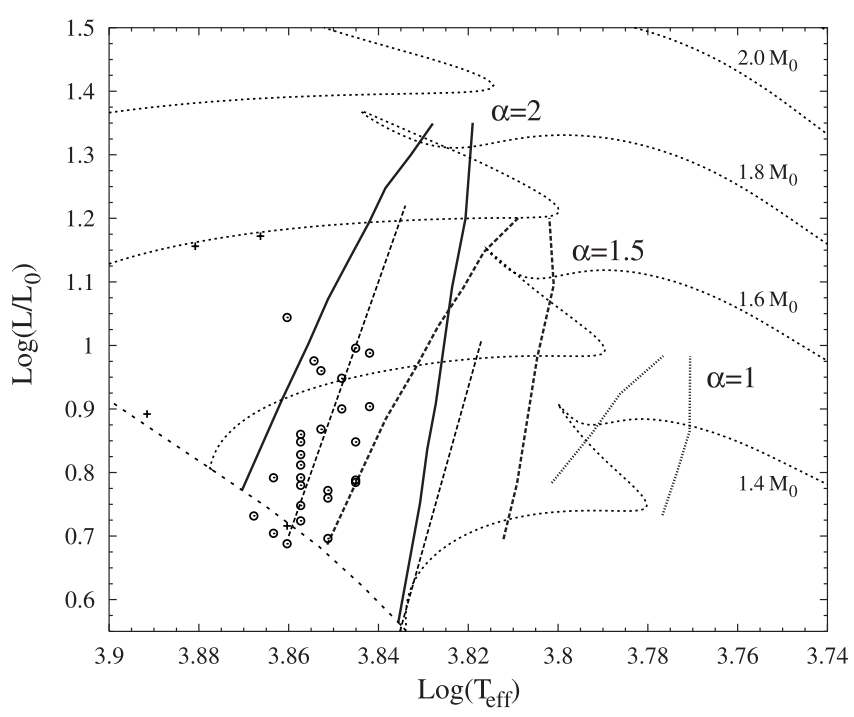

Fig. 9. $\gamma$ Dor theoretical IS for $\ell=1$ modes, for three families of models with different values of $\alpha: 1,1.5$ and 2 obtained with our TDC treatment (thick lines), compared to the FC results of Warner et al. (2003) (thin dashed lines, $\alpha=1.87$ ). The small circles correspond to observations of 27 bona fide $\gamma$ Dor stars. The crosses correspond to 3 peculiar binary stars. $\alpha=2$ theoretical IS best agree with observations.

individual ones for each mode. For any model inside the IS, at least one unstable high order g-mode is found; outside it all are found to be stable. The small circles correspond to the observed positions of 27 bona fide $\gamma$ Dor stars from the Handler (2002) catalogue; their effective temperatures are taken from Kaye et al. (1999), who used the calibrations of Villa (1998). Some evolutionary tracks for models with $\alpha=2$ are also given in this figure. The best agreement between the theoretical and observed IS is obtained for $\alpha=2$ models. Three $\gamma$ Dor stars are too hot and out of the theoretical IS: HD 209295, HD 19684 and HD 221866 denoted by crosses in Fig. 9. HD $209295\left(\log T_{\text {eff }}=3.88, \log \left(L / L_{\odot}\right)=1.16\right)$, a binary that could be tidally excited (Handler 2002), shows both long and short period pulsations (see Sect. 6). HD 19684 $\left(\log T_{\text {eff }}=3.865, \log \left(L / L_{\odot}\right)=1.17\right)$ is a spectroscopic binary similar to HD 209295 (Kaye \& Fekel 2003). HD 221866 is a double-lined spectroscopic binary whose primary is an Am star (Kaye et al. 2004); it is probably the secondary $\left(\log T_{\text {eff }}=3.86\right.$, $\log \left(L / L_{\odot}\right)=0.72$ ) that is pulsating (unique cross inside the IS) and not the primary $\left(\log T_{\text {eff }}=3.89, \log \left(L / L_{\odot}\right)=0.89\right)$. As for $\delta$ Sct stars, we see that the theoretical predictions are very sensitive to $\alpha$. There are however 2 important differences compared to the $\delta$ Sct case. First, for $\gamma$ Dor, the position of the blue edge of the IS is much more sensitive to $\alpha$ than for $\delta$ Sct, which implies that the appearance of the instability is connected with a deep enough convective envelope. We will discuss this in details in Sect. 4.3. Second, a red edge of the IS is also found in the FC case as shown by Warner et al. (2003), while this is not the case for $\delta$ Sct. Therefore, the stabilization mechanisms at the red edge of the $\delta$ Sct and $\gamma$ Dor IS must be different, as will be discussed in Sect. 4.4.

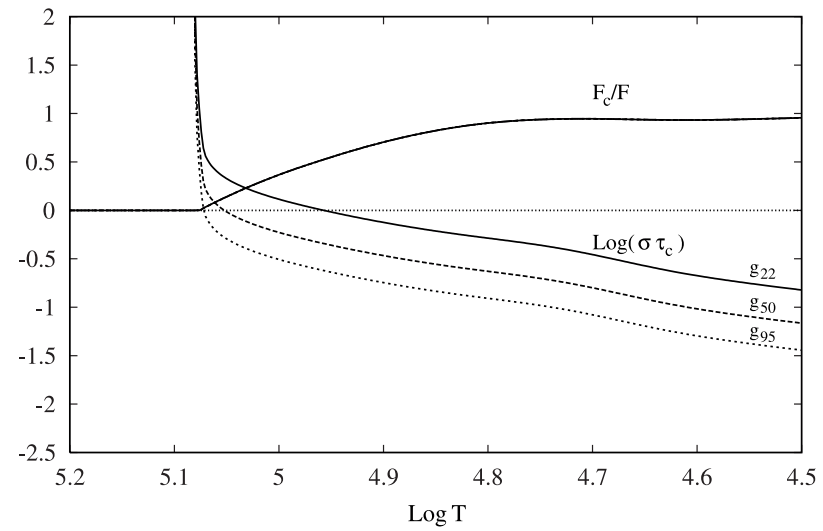

Fig. 10. $\log \left(\sigma \tau_{\mathrm{c}}\right)$ and $F_{\mathrm{c}} / F$ for the same model (Model 5 of Table 1) and 3 different $\ell=1$ g-modes: $\mathrm{g}_{22}$ (solid line), $\mathrm{g}_{50}$ (dashed line), and $\mathrm{g}_{95}$ (dotted line). $\sigma \tau_{\mathrm{c}}>1$ near the CE base only.

\subsection{Excitation mechanism}

Guzik et al. (2000) have interpreted the driving of the $\gamma$ Dor g-modes as a flux blocking mechanism occurring at the CE base. In their FC models, they neglect the Lagrangian variation of convective luminosity. Because of the radiative flux drop at the $\mathrm{CE}$ base, $\mathrm{d} \delta L_{\mathrm{R}} / \mathrm{d} m<0$ at the hot phase of pulsation, which is driving the oscillations as shown by Eq. (1).

Guzik et al. (2000) argue that FC models can be used to explain this blocking mechanism because it occurs at the $\mathrm{CE}$ base where the lifetime of convective elements is longer than the pulsation period $\left(\sigma \tau_{\mathrm{c}}>1\right)$. In Fig. 10, we show $\log \left(\sigma \tau_{\mathrm{c}}\right)$ as a function of $\log T$ for the same model but for 3 different pulsation frequencies corresponding to the modes $\mathrm{g}_{22}, \mathrm{~g}_{50}$, and $\mathrm{g}_{95}$. The zone where $\sigma \tau_{\mathrm{c}}>1$ is very thin for the low frequency modes, so that TDC models are required to test if flux blocking is really the main driving mechanism for these stars.

In Table 3, we give the values for thermal relaxation time $\tau_{\text {th }}=\int_{m}^{M} T c_{v} \mathrm{~d} m / L$ in days ( $m$ is the mass at the CE base, and $M$ is the total mass of the star), $\log T$ at the CE base and $\log (1-m / M)$ for models with different $T_{\text {eff }}$ and $\alpha$ situated at the left, middle, and right of the $\gamma$ Dor IS. In agreement with Guzik et al. (2000), we think that the position of CE base is the key point in driving $\gamma$ Dor g-modes. First, for the model at the middle of the $\gamma$ Dor IS ( $\alpha=2$ and $T_{\text {eff }}=6935 \mathrm{~K}$ ), the CE base is exactly in the transition region where the thermal relaxation time is on the same order as the g-modes pulsation periods. Driving mechanisms are thus very efficient at the CE base of this model. Secondly, for hot and hence thin CE models at the left side of the IS, the CE mass is very small, making the thermal relaxation time at the CE base very small compared to the pulsation periods, the heat capacity is too small and no significant driving or damping can occur. These are basically the physical mechanisms that explain the location of the blue edge of the $\gamma$ Dor IS and its sensitivity to the ML parameter $\alpha$.

In Fig. 11, we give the work integral obtained including $\delta \boldsymbol{F}_{\mathrm{c}}, \delta p_{\mathrm{t}}$, and $\delta \epsilon_{2}$. In order to test the validity of the flux blocking mechanism, we also give the contribution of the radial and transversal components of the radiative and convective flux perturbations on the work integral. Figure 11 shows that 


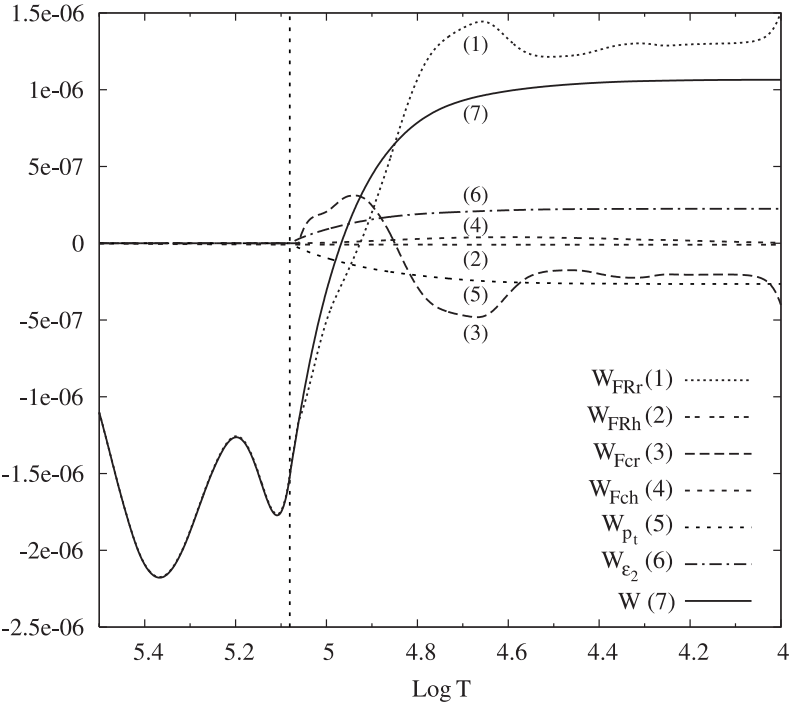

Fig. 11. Different physical components of the work integral obtained with our TDC treatment for the mode $\ell=1, g_{50}$ of Model 5 of Table 1 . Near the CE base, convective flux variations do not play a significant role, and the flux blocking mechanism dominates.

significant driving occurs at the CE base for the mode $\ell=1$, $\mathrm{g}_{50}$ (Curve 7). Decomposition in radiative and convective flux contributions shows that the convective flux variations do not play a significant role at the CE base (Curve 3). The main driving comes from the radiative flux variations (Curve 1) which supports the flux blocking mechanism proposed by Guzik et al. (2000). However the convective flux variations associated with the growth of $L_{\mathrm{c}}$ prevent $\delta L$ from dropping to zero in the TDC models when $L_{\mathrm{R}}$ goes to zero. Close to the CE base and for $\log T$ around 4.6, $\delta L_{\mathrm{c}}$ has a small destabilizing influence, but its major contribution is to stability (see Curve 3 in Fig. 11), therefore the TDC case is found to be less unstable than the FC one. We also notice small variations of $W_{\mathrm{f}_{\mathrm{R}}}$ (Curve 1 ) inside the CE, below $\log T=4.8$. This is due to $\kappa$-mechanism in the $\mathrm{He}$ and $\mathrm{H}$ partial ionization zones. However, radiative luminosity $L_{\mathrm{R}}$ is strongly reduced in these regions so that the $\kappa$-mechanism which acts through $L_{\mathrm{R}}$ is never efficient in $\gamma$ Dor stars. We see also in Fig. 11 that the transversal components of radiative and convective flux variations (Curves 2 and 4) do not play a significant role in the work integral, due to the fact that the horizontal wavelength $(r /(\ell(\ell+1)))$ is much larger than the scale heights of the different physical quantities in the superficial layers of the star. The contribution of $\delta p_{\mathrm{t}}$ and $\delta \epsilon_{2}$ on the work integral are given in Curves 5 and 6 respectively. We see that these terms have an opposite effect, so that the work integral that includes all the perturbed convection terms is close to the one with only the perturbed convective flux.

Finally, the flux blocking mechanism is also illustrated in Fig. 12, where we give $\mathfrak{R}\left\{\delta L / L_{\mathrm{s}}\right\}$ (solid line) and $2\left(\Gamma_{3}-1\right.$ ) $\mathfrak{R}\{\delta \rho / \rho\}$ (dashed line) for the same mode and model as Fig. 11. In this figure, $\delta L$ is the Lagrangian variation of the total luminosity and $L_{\mathrm{S}}$ the total luminosity at the photosphere. All eigenfunctions are normalized so that $\xi_{r} / r=1$ at the photosphere. We see significant decrease in $\delta L$ at the CE base, at the hot phase of pulsation, which clearly illustrates the flux blocking

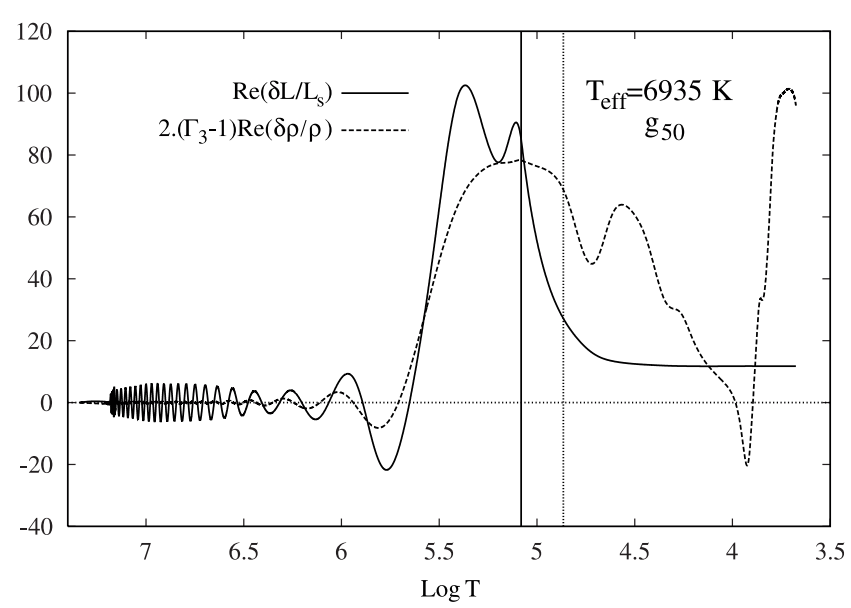

Fig. 12. $\mathfrak{R}\left\{\delta L / L_{\mathrm{s}}\right\}$ (solid line) and $2(\delta T / T)_{\mathrm{ad}} \equiv 2\left(\Gamma_{3}-1\right) \mathfrak{R}\{\delta \rho / \rho\}$ (dashed line) for the mode $\ell=1, \mathrm{~g}_{50}$, obtained with TDC treatment and Model 5 of Table 1. The vertical solid line corresponds to the $\mathrm{CE}$ base and the g-mode cavity top, and the vertical dotted line corresponds to the p-mode cavity base. Amplitudes are small for $\log T>5.6$ and large in the flux blocking region near the CE base.

Table 3. Thermal relaxation time $\left(\tau_{\text {th }}\right), \log T$ at the base of the CE and logarithm of CE mass divided by total mass, for 1.6 $M_{\odot}$ models with different $T_{\text {eff }}$ and $\alpha$.

\begin{tabular}{llllll}
\hline \hline$\alpha$ & 2 & 1 & 1.5 & 2 & 2 \\
$T_{\text {eff }}(\mathrm{K})$ & 7414 & 6932 & 6934 & 6935 & 6609 \\
\hline$\tau_{\text {th }}(\mathrm{d})$ & 0.01 & 0.007 & 0.19 & 7.7 & 1370. \\
$\log T$ & 4.50 & 4.41 & 4.74 & 5.08 & 5.51 \\
$\log (\Delta m / M)$ & -9.1 & -9.2 & -8.0 & -6.5 & -4.6 \\
\hline
\end{tabular}

mechanism. We also see that $\left(\Gamma_{3}-1\right) \delta \rho / \rho$ is large in these regions, which is needed for efficient driving (see Eq. (1)).

\subsection{Radiative damping mechanism}

As shown in Fig. 9, our TDC models predict a theoretical red edge for $\gamma$ Dor IS. Also, we can see in Fig. 8 that for the same models we predict unstable high order g-modes of $\gamma$ Dor type and unstable low order $\mathrm{p}-\mathrm{g}$ modes of $\delta$ Sct type. These modes are the same as the low order unstable g-modes shown in the middle right panel of Fig. 1. These two regions of instability are separated by a stable region corresponding to pulsation periods between 0.15 and 0.8 days (for $\ell=1$ and $\alpha=2$ ). In both cases, stabilization of the g-modes is explained by a radiative damping mechanism occurring in the g-mode cavity, defined as the region where the angular pulsation frequency is smaller than the Brunt-Väisälä and the Lamb frequencies.

We illustrate this radiative damping in Fig. 13 that gives the work integral obtained for 2 different modes and models. The bottom panel is for the mode $\ell=1 \mathrm{~g}_{70}$ of a cold model; it shows the stabilization mechanism at the red edge of the IS. The top panel is for the mode $\ell=1 \mathrm{~g}_{15}$ of a model in the middle of the $\gamma$ Dor IS and shows the stabilization mechanism for g-modes of intermediate radial order (here $\mathrm{g}_{15}$ ) between the 2 regions of instability corresponding to low order $\mathrm{p}-\mathrm{g}$ modes and high 

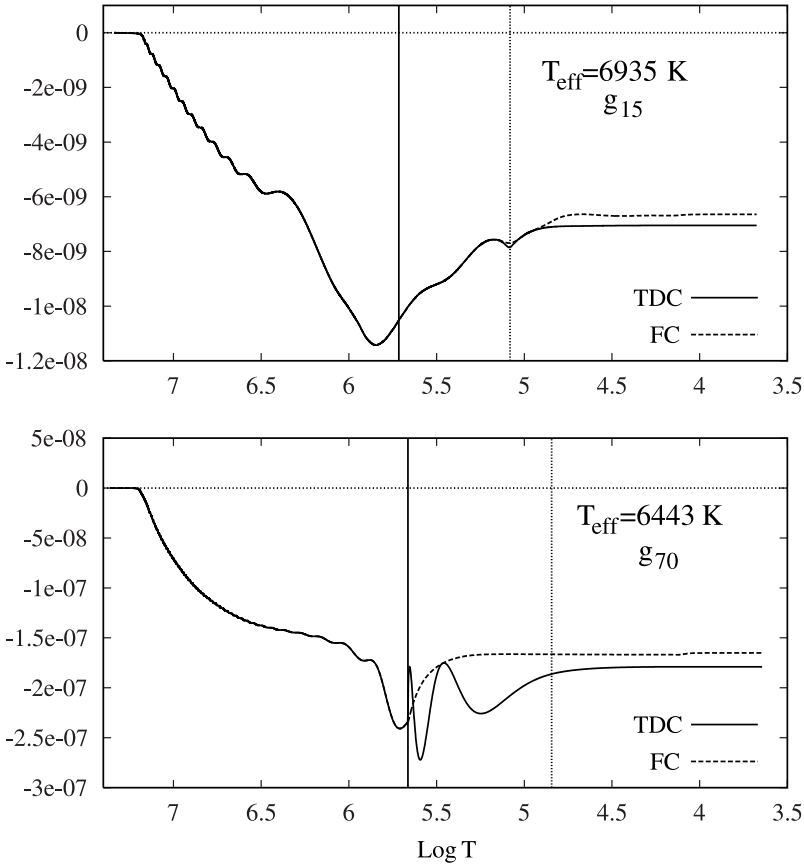

Fig. 13. Work integrals obtained with TDC and FC treatment $\ell=1$ modes. Bottom panel is for the mode $\mathrm{g}_{70}(P=2.41$ days $)$ of Model 6 of Table 1 and top panel is for the mode $\mathrm{g}_{15}(P=0.59$ days $)$ of Model 5 of Table 1 . The vertical solid line corresponds to the g-mode cavity top (and CE base in bottom panel). The vertical dotted line corresponds to the p-mode cavity base (and CE base in top panel). In both cases, significant damping occurs in the g-mode cavity.

order g-modes. In both cases, we see that the radiative damping mechanism in the g-mode cavity (between $\log T=7.2$ and $\log T=5.7)$ is much more significant than the excitation near the CE base.

The radiative damping mechanism is easily explained; since it occurs deep in the star, we can adopt the quasi-adiabatic approximation in our explanation. We use Eq. (1) of this paper and the perturbed diffusion equation (Eq. (D.6) of Paper I). As the eigenfunctions oscillate quickly in the g-mode cavity, their derivatives are much more significant than those of the equilibrium quantities; as result we can assume constant coefficients, and the term $\mathrm{d}(\delta T / T) / \mathrm{d} \ln T$ dominates in the perturbed diffusion equation. We checked that $\mathrm{d}(\delta T / T) / \mathrm{d} \ln T$ dominates in all the radiative zone for the mode $\mathrm{g}_{70}$ and from $\log T=7.2$ to $\log T=6.3$ for the mode $\mathrm{g}_{15}$. Therefore, we find in this zone:

$W \simeq-\int_{0}^{r} \frac{1}{\sigma} \frac{L}{\mathrm{~d} \ln T / \mathrm{d} r} \frac{\delta T}{T} \frac{\mathrm{d}^{2}(\delta T / T)}{\mathrm{d} r^{2}} \mathrm{~d} r$.

As $\delta T / T$ is oscillating, its second order derivative has the opposite sign, the work is negative, and the oscillations are damped.

In the following discussion, we use the notation $(\delta T / T)_{\mathrm{ad}} \equiv$ $\left(\Gamma_{3}-1\right) \mathfrak{R}\{\delta \rho / \rho\}$, and will even omit the subscript "ad" when we consider the quasi-adiabatic region. In Fig. 14, we give $\mathfrak{R}\left\{\delta L / L_{\mathrm{s}}\right\}$ (solid line) and $4(\delta T / T)_{\text {ad }}$ (dashed line) for the same modes and models as Fig. 13. $L_{\mathrm{s}}$ is the total luminosity at the photosphere. For the sake of clarity in this figure, we only show results for TDC models, but we notice that the results for FC models are very similar. Comparing this figure with Fig. 12 (note that $(\delta T / T)_{\mathrm{ad}}$ is multiplied by 2 in Fig. 12) allows us
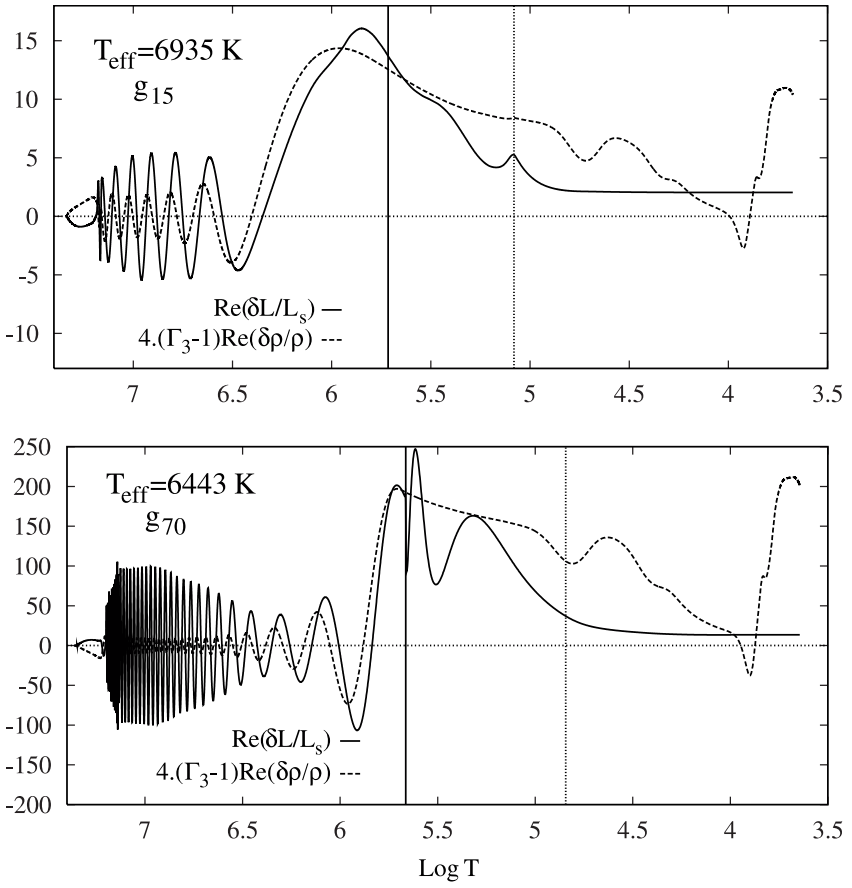

Fig. 14. $\mathfrak{R}\left\{\delta L / L_{\mathrm{s}}\right\}$ (solid line) and $4(\delta T / T)_{\mathrm{ad}} \equiv 4\left(\Gamma_{3}-1\right) \mathfrak{R}\{\delta \rho / \rho\}$ (dashed line), for TDC models and $\ell=1$ modes. The bottom panel is for mode $g_{70}$ of Model 6 of Table 1, and the top panel is for mode $\mathrm{g}_{15}$ of Model 5 of Table 1. Vertical lines have the same meaning as in Fig. 13. The spatial oscillations of the eigenfunctions have significant amplitudes in the g-mode cavity.

to understand why the radiative damping dominates in some cases, while the flux blocking driving dominates in other cases. In Fig. 14, $|\delta T / T|$ is significant in the g-mode cavity compared to its values in more superficial layers. Therefore, radiative damping in the g-mode cavity is very efficient and gives these modes their stability. In contrast, for high order g-modes of a model in the middle of the $\gamma$ Dor IS (Fig. 12), $|\delta T / T|$ is very small in the g-mode cavity compared to the amplitudes at the CE base, so that the flux blocking driving at the CE base is more efficient than the radiative damping. Finally, radiative damping is efficient when the eigenfunctions oscillate quickly in the g-mode cavity, leading to large values of $\mathrm{d}^{2}(\delta T / T) / \mathrm{d} r^{2}$ in Eq. (7). This is of course not the case for $\delta$ Sct type low order p-modes, which explains why we find such unstable p-modes in our models.

It is not easy to interpret what determines the eigenfunction amplitudes in different layers of the star, a study beyond the scope of this paper. We only mention that the location of the upper boundary of the g-mode cavity (depending on the frequency) certainly plays an important role. For mode $g_{15}$ of Model 5 in Table 1, the g-mode cavity upper boundary is deep in the $\operatorname{star}($ at $\log T=5.71)$ and given by intersection with the Lamb frequency. For mode $\mathrm{g}_{50}$ of the same model, the top of the g-mode cavity is close to the surface at the CE base ( $\log T=5.08)$. Finally, for the mode $g_{70}$ of Model 6 of Table 1 , the top of the g-mode cavity is at the CE base, which is deep for this colder model (at $\log T=5.67$ ). For the 2 cases in which the top of the g-mode cavity is deep in the star, amplitudes of the eigenfunctions are larger in the g-mode cavity compared to 
those in the more superficial layers (Fig. 14). This is the opposite for the mode $\mathrm{g}_{50}$, and amplitudes are much larger near the CE base than in the g-mode cavity (Fig. 12).

The relative importance of radiative damping in the g-mode cavity compared to excitation due to the flux blocking thus explains the position of the $\gamma$ Dor IS red edge and the stability of intermediate radial order g-modes in the models with both unstable high order g-modes and unstable low order $\mathrm{p}-\mathrm{g}$ modes. In particular, we have seen that stabilization of the $\gamma$ Dor high order g-modes is efficient for models with a given depth of the CE, which explains the high sensitivity of the red edge location with respect to the ML parameter $\alpha$.

We still have to explain the driving occurring between $\log T=5.8$ and $\log T=5.2$ for mode $\mathrm{g}_{15}$ (top panel of Fig. 13). In this region, $\mathrm{d}(\delta T / T) / \mathrm{d} \ln T$ no longer dominates in the perturbed diffusion equation, and the driving is caused by a classical $\kappa$-mechanism occurring in the partial ionization zone of Iron, as in $\beta$ Cep and Slowly Pulsating B stars. The same $\kappa$-driving can be seen for mode $\mathrm{g}_{50}$ between $\log T=5.4$ and $\log T=5.2$ (Fig. 11).

Concerning other marginal driving and damping mechanisms, we notice first that the $\kappa$-mechanism in the $\mathrm{He}$ and $\mathrm{H}$ partial ionization zones can play a role in FC models. Secondly, Fig. 11 (Curve 3) shows that some damping can occur in our TDC models due to the convective flux variations, which completely cancels the above-mentioned $\kappa$-mechanism inside the CE. However, all these effects are always smaller than the two main mechanisms in $\gamma$ Dor models: the flux blocking driving at the $\mathrm{CE}$ base and radiative damping in the g-mode cavity. The balance between these two mechanisms explains the location of the $\gamma$ Dor IS.

To summarize, the g-mode cavity always has a stabilizing influence, while the flux blocking mechanism at the bottom of the CE has a destabilizing one. The balance between these two mechanisms is a function of the amplitude of $\delta T / T$ in the g-mode cavity compared to the bottom of the CE. Depending on the behaviour of $\delta T / T$ the mode will be unstable or stable. For low enough order g-modes, the excitation between the top of the g-mode cavity and the bottom of the CE cannot, however, be neglected. Radiative damping explains the existence of the $\gamma$ Dor IS red edge and of a stable region in the period domain between the unstable high order g-modes and low order $\mathrm{p}-\mathrm{g}$ modes. However the blue edge of the $\gamma$ Dor IS is due to the breakdown of the adiabatic approximation already below the CE base, which implies less efficient flux blocking at the $\mathrm{CE}$ base and more stabilization just below it.

\section{Influence of the TDC treatment on the frequencies and growth rates}

Perturbation of convective flux, turbulent pressure and dissipation rate of turbulent kinetic energy into heat are the different terms of our TDC treatment that influence frequencies and growth rates. Table 4 gives the results obtained for a $\delta$ Sct and a $\gamma$ Dor model. The selected modes correspond to boundaries of the instability domain.

The differences between adiabatic and FC non-adiabatic frequencies are very small for all the modes. For all the $\gamma$ Dor g-modes and for the $\delta$ Sct modes with low frequency, the differences between FC and TDC non-adiabatic frequencies are even smaller. We conclude that for $\delta$ Sct and $\gamma$ Dor stars, an adiabatic analysis gives precise enough pulsation frequencies.

However, only a non-adiabatic analysis can give the imaginary part of $\omega$ (dimensionless damping rate). Considering the last three colums of Table 4, we see that when all the TDC terms are taken into account (Col. 11), the damping rates are not significantly different from those obtained when only the convective flux perturbation (Col. 9) is taken into account. This result is obtained because the effects of the turbulent pressure perturbation (Col. 10) and of the turbulent kinetic energy dissipation on the damping rates nearly compensate in agreement with Ledoux \& Walraven (1958) and Eqs. (3) and (4). Therefore, the IS obtained taking all the convection terms into account are not expected to be very different from those that only include perturbation of the convective flux. The theoretical IS are much more sensitive to the value of the ML parameter $\alpha$, as shown in Figs. 2, 4, and 9.

\section{6. $\delta$ Sct and $\gamma$ Dor simultaneous pulsations}

Early on, when the $\gamma$ Dor new class of pulsating stars was discovered, a question was raised about the relationship between them and the $\delta$ Sct stars because of their location in the HR diagram (Breger \& Beichbuchner 1996). Stars pulsating simultaneously with $\delta$ Sct p-modes and $\gamma$ Dor g-modes have been sought (Handler \& Shobbrook 2002), and one star with both types of modes was found: HD 209295. However, this star is very peculiar as it is a close binary, and the long period modes could be tidally excited (Handler et al. 2002).

Although search for such hybrid $\delta$ Sct- $\gamma$ Dor stars has not been fully conclusive until now, we stress that their existence is predicted by our theoretical models. More precisely, in Fig. 8 we see that those models with unstable long period gravity modes also have unstable short period pressure modes; only the unstable modes with $Q \geq 0.04$ days are given in Fig. 8 . These short period unstable modes are the $\delta$ Sct type modes analysed in Sect. 3. In Fig. 15, we give both the theoretical $\gamma$ Dor IS and the $\delta$ Sct IS red edge for $\alpha=1.8$ models. We see that a significant part of the $\gamma$ Dor IS is inside the $\delta$ Sct one. All the theoretical models in this intersection have both types of unstable modes. Handler \& Shobbrook (2002) showed that $\delta$ Sct and $\gamma$ Dor stars have very different ranges for the pulsation constant $Q=P t_{\mathrm{dyn}, \odot} / t_{\mathrm{dyn}}$, without any intermediate cases, which is in perfect agreement with our theoretical results. As we see in Fig. 8, the unstable zones of high order g-mode and low order $\mathrm{p}$-modes are clearly separated by a stable zone explained by the effect of radiative damping (Sect. 4.4). Table 5 gives the complete range of $Q$-values that we find for each of these unstable zones for the same model with both $\delta$ Sct-type and $\gamma$ Dor-type unstable modes. Our theoretical values are in perfect agreement with observations by Handler \& Shobbrook (2002). We conclude that there are no theoretical arguments against the existence of hybrid $\delta$ Sct- $\gamma$ Dor stars; on the contrary, it is very important to continue to search for such stars, which would be of considerable interest for asteroseismology, 
Table 4. Frequencies $(f=\mathfrak{R}\{\sigma\} /(2 \pi))$ and dimensionless damping rates $\left(\mathfrak{J}\{\omega\}=\mathfrak{J}\{\sigma\} t_{\text {dyn }}\right)$ for different modes of a $\delta$ Sct model (top) and a $\gamma$ Dor model (bottom). Column 3 gives the adiabatic frequency (in $\mu \mathrm{Hz}$ ). Column 4 gives the difference between the non-adiabatic frequencies of FC models and the adiabatic frequencies. Columns 5 to 7 give the difference between the non-adiabatic frequencies of TDC models and the adiabatic frequencies. Columns 8 to 11 give the dimensionless damping rate of FC models (Col. 8) and of TDC models (Cols. 9 to 11); it is negative for unstable modes and positive for stable modes. The perturbation of convective flux $\delta \boldsymbol{F}_{\mathrm{c}}$ is taken into account in all the TDC models. The perturbation of turbulent pressure $\delta p_{t}$ is taken into account in Cols. 6, 7, 10 and 11. The perturbation of turbulent kinetic energy dissipation $\delta \epsilon_{2}$ is taken into account in Cols. 7 and 11 .

\begin{tabular}{|c|c|c|c|c|c|c|c|c|c|c|}
\hline $\begin{array}{c}\ell \\
(1)\end{array}$ & $\begin{array}{c}n \\
(2)\end{array}$ & $\begin{array}{l}f_{\text {ad }} \\
(3)\end{array}$ & $\begin{array}{c}\Delta f_{\mathrm{FC}} \\
(4)\end{array}$ & $\begin{array}{c}\Delta f_{\delta F_{\mathrm{c}}} \\
(5)\end{array}$ & $\begin{array}{c}\Delta f_{\delta p_{\mathrm{t}}} \\
(6)\end{array}$ & $\begin{array}{c}\Delta f_{\delta\left(p_{\mathrm{t}}, \epsilon_{2}\right)} \\
\quad(7)\end{array}$ & $\begin{array}{c}\mathfrak{J}\left(\omega_{\mathrm{FC}}\right) \\
(8)\end{array}$ & $\begin{array}{c}\mathfrak{J}\left(\omega_{\delta F_{\mathrm{c}}}\right) \\
(9)\end{array}$ & $\begin{array}{c}\mathfrak{J}\left(\omega_{\delta p_{\mathrm{t}}}\right) \\
(10)\end{array}$ & $\begin{array}{c}\mathfrak{J}\left(\omega_{\delta\left(p_{1}, \epsilon_{2}\right)}\right) \\
(11)\end{array}$ \\
\hline \multicolumn{11}{|c|}{$\delta$ Sct: Model 7 of Table $1, t_{\mathrm{dyn}}=3460 \mathrm{~s}$} \\
\hline \multirow[t]{2}{*}{0} & 1 & $1.631 \mathrm{E}+02$ & $2.322 \mathrm{E}-02$ & $2.321 \mathrm{E}-02$ & $2.324 \mathrm{E}-02$ & $2.322 \mathrm{E}-02$ & $-6.449 \mathrm{E}-07$ & $-7.699 \mathrm{E}-07$ & $-9.807 \mathrm{E}-07$ & $-8.291 \mathrm{E}-07$ \\
\hline & 4 & $3.125 \mathrm{E}+02$ & $1.214 \mathrm{E}-02$ & $-6.325 \mathrm{E}-03$ & $-7.519 \mathrm{E}-04$ & $-3.993 \mathrm{E}-03$ & $-9.658 \mathrm{E}-05$ & $-3.475 \mathrm{E}-05$ & $-1.192 \mathrm{E}-04$ & $-3.061 \mathrm{E}-05$ \\
\hline \multirow[t]{2}{*}{1} & -2 & $1.197 \mathrm{E}+02$ & $2.765 \mathrm{E}-02$ & $2.765 \mathrm{E}-02$ & $2.765 \mathrm{E}-02$ & $2.765 \mathrm{E}-02$ & $-1.170 \mathrm{E}-09$ & $-9.845 \mathrm{E}-10$ & $-6.680 \mathrm{E}-10$ & $-6.906 \mathrm{E}-10$ \\
\hline & 3 & $2.723 \mathrm{E}+02$ & $9.861 \mathrm{E}-03$ & $4.281 \mathrm{E}-03$ & $6.390 \mathrm{E}-03$ & $5.196 \mathrm{E}-03$ & $-3.894 \mathrm{E}-05$ & $-2.907 \mathrm{E}-05$ & $-6.623 \mathrm{E}-05$ & $-3.432 \mathrm{E}-05$ \\
\hline \multirow[t]{2}{*}{2} & -4 & $1.081 \mathrm{E}+02$ & $1.372 \mathrm{E}-02$ & $1.372 \mathrm{E}-02$ & $1.372 \mathrm{E}-02$ & $1.372 \mathrm{E}-02$ & $-1.021 \mathrm{E}-09$ & $-7.092 \mathrm{E}-10$ & $-1.555 \mathrm{E}-10$ & $-2.415 \mathrm{E}-10$ \\
\hline & 2 & $3.005 \mathrm{E}+02$ & $7.918 \mathrm{E}-03$ & $-4.078 \mathrm{E}-03$ & $-1.708 \mathrm{E}-04$ & $-2.358 \mathrm{E}-03$ & $-6.793 \mathrm{E}-05$ & $-3.305 \mathrm{E}-05$ & $-8.948 \mathrm{E}-05$ & $-2.932 \mathrm{E}-05$ \\
\hline \multirow[t]{2}{*}{3} & -5 & $1.205 \mathrm{E}+02$ & $1.919 \mathrm{E}-02$ & $1.919 \mathrm{E}-02$ & $1.919 \mathrm{E}-02$ & $1.919 \mathrm{E}-02$ & $-2.942 \mathrm{E}-09$ & $-2.611 \mathrm{E}-09$ & $-1.329 \mathrm{E}-09$ & $-1.364 \mathrm{E}-09$ \\
\hline & 2 & $3.172 \mathrm{E}+02$ & $5.659 \mathrm{E}-03$ & $-1.603 \mathrm{E}-02$ & $-9.262 \mathrm{E}-03$ & $-1.296 \mathrm{E}-02$ & $-1.106 \mathrm{E}-04$ & $-3.456 \mathrm{E}-05$ & $-1.137 \mathrm{E}-04$ & $-1.155 \mathrm{E}-05$ \\
\hline \multicolumn{11}{|c|}{$\gamma$ Dor: Model 5 of Table $1, t_{\mathrm{dyn}}=3800 \mathrm{~s}$} \\
\hline \multirow[t]{3}{*}{1} & -82 & $3.811 \mathrm{E}+00$ & $2.039 \mathrm{E}-04$ & $-2.608 \mathrm{E}-04$ & $-2.760 \mathrm{E}-04$ & $-2.619 \mathrm{E}-04$ & $-1.855 \mathrm{E}-06$ & $2.166 \mathrm{E}-07$ & $5.067 \mathrm{E}-07$ & $-5.007 \mathrm{E}-08$ \\
\hline & -22 & $1.365 \mathrm{E}+01$ & $3.082 \mathrm{E}-03$ & $3.082 \mathrm{E}-03$ & $3.082 \mathrm{E}-03$ & $3.082 \mathrm{E}-03$ & $-6.447 \mathrm{E}-09$ & $-6.134 \mathrm{E}-10$ & $3.925 \mathrm{E}-09$ & $-1.545 \mathrm{E}-09$ \\
\hline & -2 & $1.181 \mathrm{E}+02$ & $3.343 \mathrm{E}-02$ & $3.343 \mathrm{E}-02$ & $3.343 \mathrm{E}-02$ & $3.343 \mathrm{E}-02$ & $-7.644 \mathrm{E}-09$ & $-8.936 \mathrm{E}-09$ & $-1.097 \mathrm{E}-08$ & $-9.551 E-09$ \\
\hline \multirow[t]{3}{*}{2} & -94 & $5.752 \mathrm{E}+00$ & $-1.270 \mathrm{E}-03$ & $-1.729 \mathrm{E}-03$ & $-1.746 \mathrm{E}-03$ & $-1.709 \mathrm{E}-03$ & $-1.289 \mathrm{E}-06$ & $6.185 \mathrm{E}-07$ & $8.926 \mathrm{E}-07$ & $-1.631 \mathrm{E}-07$ \\
\hline & -23 & $2.251 \mathrm{E}+01$ & $4.231 \mathrm{E}-03$ & $4.230 \mathrm{E}-03$ & $4.230 \mathrm{E}-03$ & $4.230 \mathrm{E}-03$ & $-1.010 \mathrm{E}-08$ & $-2.919 \mathrm{E}-09$ & $1.291 \mathrm{E}-08$ & $-4.308 \mathrm{E}-09$ \\
\hline & -4 & $1.065 \mathrm{E}+02$ & $1.800 \mathrm{E}-02$ & $1.800 \mathrm{E}-02$ & $1.800 \mathrm{E}-02$ & $1.800 \mathrm{E}-02$ & $-5.679 \mathrm{E}-09$ & $-5.231 \mathrm{E}-09$ & $-6.481 \mathrm{E}-09$ & $-4.628 \mathrm{E}-09$ \\
\hline \multirow[t]{3}{*}{3} & -99 & $7.730 \mathrm{E}+00$ & $-1.274 \mathrm{E}-03$ & $-1.687 \mathrm{E}-03$ & $-1.693 \mathrm{E}-03$ & $-1.631 \mathrm{E}-03$ & $-8.644 \mathrm{E}-07$ & $6.347 \mathrm{E}-07$ & $1.058 \mathrm{E}-06$ & $-4.975 \mathrm{E}-07$ \\
\hline & -26 & $2.852 \mathrm{E}+01$ & $3.816 \mathrm{E}-03$ & $3.814 \mathrm{E}-03$ & $3.813 \mathrm{E}-03$ & $3.814 \mathrm{E}-03$ & $-3.132 \mathrm{E}-08$ & $-9.095 \mathrm{E}-09$ & $4.206 \mathrm{E}-08$ & $-9.212 \mathrm{E}-09$ \\
\hline & -6 & $1.071 \mathrm{E}+02$ & $1.733 \mathrm{E}-02$ & $1.733 \mathrm{E}-02$ & $1.733 \mathrm{E}-02$ & $1.733 \mathrm{E}-02$ & $-2.221 \mathrm{E}-09$ & $-2.226 \mathrm{E}-09$ & $-2.774 \mathrm{E}-09$ & $-1.702 \mathrm{E}-09$ \\
\hline
\end{tabular}

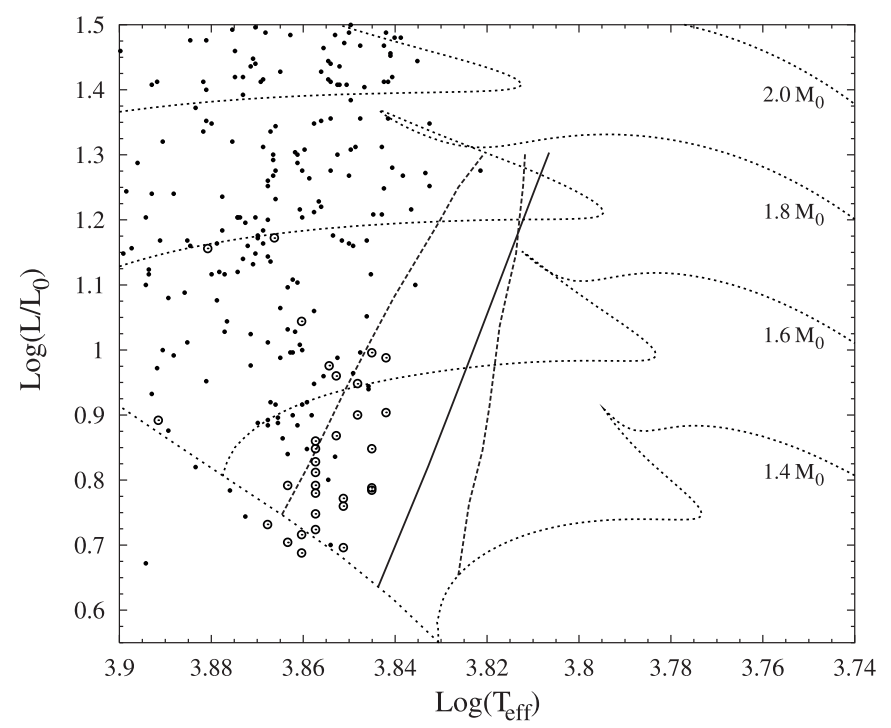

Fig. 15. $\gamma$ Dor theoretical IS for $\ell=1$ modes (dashed lines) and $\delta$ Sct red edge of the IS for the fundamental radial mode (solid line), for $\alpha=1.8$ models. The small empty circles correspond to observations of bona fide $\gamma$ Dor stars and the small full points correspond to observations of $\delta$ Sct stars. Theoretical models in the intersection between the two IS have both $\gamma$ Dor type unstable g-modes and $\delta$ Sct type unstable p-modes. with both high order g-modes probing the deep interior and pmodes probing the more superficial layers.

\section{Conclusion}

We included the TDC treatment presented in Paper I in our nonradial non-adiabatic pulsation code and applied it to the study of the driving and damping mechanisms in $\delta$ Sct and $\gamma$ Dor stars. For $\delta$ Sct stars, we succeed in reproducing both the blue and red edges for radial, as well as for non-radial modes. We also obtained theoretical IS for the $\gamma$ Dor g-modes, and showed that the theoretical IS of both $\delta$ Sct and $\gamma$ Dor stars are very sensitive to the value of the ML parameter $\alpha$. We get good agreement with observations for models with $\alpha$ between 1.8 (solar calibrated value) and 2 . We analysed the effect of the different terms of our TDC treatment on the frequencies and on the driving and damping mechanisms. The difference between adiabatic and non-adiabatic frequencies is very small for these stars. Turbulent pressure perturbation $\left(\delta p_{\mathrm{t}}\right)$ has a driving effect in our $\delta$ Sct models and a damping effect in our $\gamma$ Dor models. The turbulent kinetic energy dissipation perturbation $\left(\delta \epsilon_{2}\right)$ has the opposite effect of $\delta p_{\mathrm{t}}$ on the driving and damping. Hence, results taking $\delta F_{\mathrm{c}}, \delta p_{\mathrm{t}}$, and $\delta \epsilon_{2}$ into account are similar to results taking only $\delta F_{\mathrm{c}}$ into account. For $\gamma$ Dor stars, 
Table 5. Maximal and minimal $Q$-values (in days) of unstable $\delta$ Sct-type and $\gamma$ Dor-type modes for the same model (Model 8 of Table 1).

\begin{tabular}{llllll}
\hline \hline & & $\ell=0$ & $\ell=1$ & $\ell=2$ & $\ell=3$ \\
\hline \multirow{2}{*}{ Dor } & $Q_{\max }(\mathrm{d})$ & & 0.907 & 0.593 & 0.451 \\
& $Q_{\min }(\mathrm{d})$ & & 0.387 & 0.232 & 0.170 \\
\hline \multirow{3}{*}{ Sct } & $Q_{\max }(\mathrm{d})$ & 0.033 & 0.041 & 0.046 & 0.041 \\
& $Q_{\min }(\mathrm{d})$ & 0.020 & 0.020 & 0.020 & 0.019 \\
\hline
\end{tabular}

we have shown that $\delta F_{\mathrm{c}}$ has no significant effect on the damping and/or driving at the CE base. Hence, our TDC models show that flux blocking at the CE base is the main driving mechanism of $\gamma$ Dor g-modes. Finally, our theoretical models predict the possible occurrence of simultaneous $\delta$ Sct p-modes and $\gamma$ Dor g-modes oscillations for the same stellar model. The discovery of such hybrid $\delta$ Sct $-\gamma$ Dor stars would be of particular interest for asteroseismology. Observed high-order g-modes would allow us to probe their deep interior, while the observed p-modes would allow us to probe the more superficial layers, all of which means that search should be intensified. We intend to include the perturbation of non-diagonal components of the Reynolds stress tensor in our non-adiabatic code, following the theory of Gabriel (1987), and to study its influence on the driving of $\delta$ Sct and $\gamma$ Dor non-radial modes. Application of our TDC treatment to solar-type oscillations will also be presented in a future paper.

Acknowledgements. M.A.D. acknowledges support through a European Community Marie Curie Fellowship. A.G. and R.G. acknowledge financial support from the program ESP2001-4528-PE.

\section{References}

Alexander, D. R., \& Ferguson, J. W. 1994, ApJ, 437, 879

Baker, N., \& Kippenhahn, R. 1965, ApJ, 142, 868

Balmforth, N. J. 1992, MNRAS, 255, 603

Breger, M., \& Beichbuchner, F. 1996, A\&A, 313, 851

Christensen-Dalsgaard, J., \& Däppen, W. 1992, A\&ARv, 4, 267
Dupret, M.-A. 2002, Ph.D. Thesis, Bull. Soc. Roy. Sc. Liège, 5-6, 249

Dupret, M.-A., Grigahcène, A., Garrido, R., et al. 2004a, A\&A, 414, L17

Dupret, M.-A., Montalban, J., Grigahcène, A., et al. 2004b, in Variable Stars in the Local Group, ed. D. W. Kurtz, \& K. Pollard, PASP Conf. Ser., 310, 470

Gabriel, M. 1987, A\&A, 175, 125

Gonczi, G. 1986, A\&A, 157, 133

Gough, D. O. 1977, ApJ, 214, 196

Grigahcène, A., Dupret, M.-A., Garrido, R., et al. 2004a, in JENAM Minisymp. asteroseismology, ed. Z. Kollath, \& G. Handler, CoAst, 145, 9

Grigahcène, A., Dupret, M.-A., Gabriel, M., et al. 2004b, A\&A, in press (Paper I)

Guzik, J. A., Kaye, A. B., Bradley, P. A., et al. 2000, ApJ, 542, L57

Handler, G., \& Shobbrook, R. R. 2002, MNRAS, 333, 251

Handler, G. 2002, http://www . astro.univie.ac.at/ dsn/ gerald/gdorlist.html

Handler, G., Balona, L. A., Shobbrook, R. R., et al. 2002, MNRAS, 333,262

Houdek, G. 2000, in The 6th Vienna Workshop on $\delta$ Scuti and related stars, ed. M. Montgommery, \& M. Breger, PASP Conf. Ser., 210, 454

Iglesias, C. A., \& Rogers, F. J. 1996, ApJ, 464, 943

Kaye, A. B., Handler, G., Krisciunas, K., et al. 1999, PASP, 111, 840

Kaye, A. B., \& Fekel, F. C. 2003, IBVS, 5465, 1

Kaye, A. B., Gray, R. O., \& Griffin, R. F. 2004, PASP, 116, 558

Kupka, F., \& Montgomery, M. H. 2002, MNRAS, 330, L6

Kurucz, R. L. 1998, http://cfaku5 . harvard.edu/grids.html

Ledoux, P., \& Walraven, T. 1958, Handbuch der Physik, ed. S. Flügge, 51,353

Moon, T. T., \& Dworetsky, M. M. 1985, MNRAS, 217, 305

Pamyatnykh, A. A. 2000, in The 6th Vienna Workshop on $\delta$ Scuti and related stars, ed. M. Montgommery, \& M. Breger, PASP Conf. Ser., 210, 215

Rodriguez, E., Lopez-Gonzalez, M. J., \& Lopez de Coca, P. 2000, A\&AS, 144, 469

Villa, P. 1998, M. Sc. thesis, Univ. Vienna

Warner, P. B., Kaye, A. B., \& Guzik, J. A. 2003, ApJ, 593, 1049

Xiong, D. R., Cheng, Q. L., \& Deng, L. 1997, ApJS, 108, 529

Xiong, D. R., \& Deng, L. 2001, MNRAS, 324, 243

Xu, Y., Li, Z., Deng, L., \& Xiong, D. R. 2002, Chin. J. Astron. Astrophys., 2, 441 